\title{
Accelerated FRW solutions in Chern-Simons gravity
}

\author{
Mauricio Cataldo ${ }^{1, a}$, Juan Crisóstomo ${ }^{2, b}$, Sergio del Campo ${ }^{3, c}$, Fernando Gómez $^{2, d}$, Cristian C. Quinzacara ${ }^{2,4, e}$, \\ Patricio Salgado ${ }^{2, \mathrm{f}}$ \\ ${ }^{1}$ Departamento de Física, Universidad del Bío-Bío, Casilla 5-C, Concepción, Chile \\ ${ }^{2}$ Departamento de Física, Universidad de Concepción, Casilla 160-C, Concepción, Chile \\ ${ }^{3}$ Instituto de Física, Pontificia Universidad Católica de Valparaíso, Av. Universidad 300, Campus Curauma, Valparaíso, Chile \\ ${ }^{4}$ Facultad de Ingeniería y Tecnología, Universidad San Sebastián, Campus Las Tres Pascualas, Lientur 1457, Concepción, Chile
}

Received: 23 January 2014 / Accepted: 12 September 2014 / Published online: 15 October 2014

(C) The Author(s) 2014. This article is published with open access at Springerlink.com

\begin{abstract}
We consider a five-dimensional Einstein-ChernSimons action which is composed of a gravitational sector and a sector of matter where the gravitational sector is given by a Chern-Simons gravity action instead of the EinsteinHilbert action and where the matter sector is given by the socalled perfect fluid. It is shown that (i) the Einstein-ChernSimons (EChS) field equations subject to suitable conditions can be written in a similar way to the Einstein-Maxwell field equations; (ii) these equations have solutions that describe an accelerated expansion for the three possible cosmological models of the universe, namely, spherical expansion, flat expansion, and hyperbolic expansion when $\alpha$, a parameter of the theory, is greater than zero. This result allows us to conjecture that these solutions are compatible with the era of dark energy and that the energy-momentum tensor for the field $h^{a}$, a bosonic gauge field from the Chern-Simons gravity action, corresponds to a form of positive cosmological constant. It is also shown that the EChS field equations have solutions compatible with the era of matter: (i) In the case of an open universe, the solutions correspond to an accelerated expansion $(\alpha>0)$ with a minimum scale factor at initial time that, when time goes to infinity, the scale factor behaves as a hyperbolic sine function. (ii) In the case of a flat universe, the solutions describe an accelerated expansion whose scale factor behaves as an exponential function of time. (iii) In the case of a closed universe there is found only one solution for a universe in expansion, which behaves as a hyperbolic cosine function of time.
\end{abstract}

\footnotetext{
a e-mail: mcataldo@ubiobio.cl

b e-mail: jcrisostomo@udec.cl

c e-mail: sdelcamp@ucv.cl

de-mail: fernagomez@udec.cl

e e-mail: cristian.cortesq@uss.cl

fe-mail: pasalgad@udec.cl
}

\section{Introduction}

Some time ago it was shown that standard, five-dimensional General Relativity can be obtained from Chern-Simons gravity theory for a certain Lie algebra $\mathfrak{B}[1]$, whose generators $\left\{\boldsymbol{J}_{a b}, \boldsymbol{P}_{a}, \boldsymbol{Z}_{a b}, \boldsymbol{Z}_{a}\right\}$ satisfy the commutation relationships

$\left[\boldsymbol{J}_{a b}, \boldsymbol{J}_{c d}\right]=\eta_{a d} \boldsymbol{J}_{b c}-\eta_{a c} \boldsymbol{J}_{b d}+\eta_{b c} \boldsymbol{J}_{a d}-\eta_{b d} \boldsymbol{J}_{a c}$,

$\left[\boldsymbol{P}_{a}, \boldsymbol{J}_{b c}\right]=\eta_{a b} \boldsymbol{P}_{c}-\eta_{a c} \boldsymbol{P}_{b}$,

$\left[\boldsymbol{J}_{a b}, \boldsymbol{Z}_{c d}\right]=\eta_{a d} \boldsymbol{Z}_{b c}-\eta_{a c} \boldsymbol{Z}_{b d}+\eta_{b c} \boldsymbol{Z}_{a d}-\eta_{b d} \boldsymbol{Z}_{a c}$,

$\left[\boldsymbol{Z}_{a}, \boldsymbol{J}_{b c}\right]=\eta_{a b} \boldsymbol{Z}_{c}-\eta_{a c} \boldsymbol{Z}_{b}$

$\left[\boldsymbol{P}_{a}, \boldsymbol{P}_{b}\right]=\boldsymbol{Z}_{a b}$,

$\left[\boldsymbol{P}_{a}, \boldsymbol{Z}_{b c}\right]=\eta_{a b} \boldsymbol{Z}_{c}-\eta_{a c} \boldsymbol{Z}_{b}$

This algebra was obtained from the anti-de Sitter (AdS) algebra and a particular semigroup $S$ by means of the S-expansion procedure introduced in Refs. [2,3].

In order to write down a Chern-Simons Lagrangian for the $\mathfrak{B}$ algebra, we start from the one-form gauge connection

$\boldsymbol{A}=\frac{1}{2} \omega^{a b} \boldsymbol{J}_{a b}+\frac{1}{l} e^{a} \boldsymbol{P}_{a}+\frac{1}{2} k^{a b} \boldsymbol{Z}_{a b}+\frac{1}{l} h^{a} \boldsymbol{Z}_{a}$,

and the two-form curvature

$$
\begin{aligned}
\boldsymbol{F}= & \frac{1}{2} R^{a b} \boldsymbol{J}_{a b}+\frac{1}{l} T^{a} \boldsymbol{P}_{a}+\frac{1}{2}\left(\mathrm{D}_{\omega} k^{a b}+\frac{1}{l^{2}} e^{a} e^{b}\right) \boldsymbol{Z}_{a b} \\
& +\frac{1}{l}\left(\mathrm{D}_{\omega} h^{a}+k_{b}^{a} e^{b}\right) \boldsymbol{Z}_{a} .
\end{aligned}
$$

Consistency with the dual procedure of S-expansion in terms of the Maurer-Cartan forms [3] demands that $h^{a}$ inherits the unit of length from the fünfbein; that is why it is necessary to introduce the $l$ parameter again, this time associated with $h^{a}$.

It is interesting to observe that $\boldsymbol{J}_{a b}$ are still Lorentz generators, but $\boldsymbol{P}_{a}$ are no longer AdS boosts; in fact, $\left[\boldsymbol{P}_{a}, \boldsymbol{P}_{b}\right]=$ $\boldsymbol{Z}_{a b}$. However, $e^{a}$ still transforms as a vector under Lorentz 
transformations, as it must, in order to recover gravity in this scheme.

A Chern-Simons Lagrangian in $d=5$ dimensions is defined to be the following local function of a one-form gauge connection $\boldsymbol{A}$ :

$L_{\mathrm{ChS}}^{(5)}(\boldsymbol{A})=k\left\langle\boldsymbol{A} \boldsymbol{F}^{2}-\frac{1}{2} \boldsymbol{A}^{3} \boldsymbol{F}+\frac{1}{10} \boldsymbol{A}^{5}\right\rangle$,

where $\langle\cdots\rangle$ denotes an invariant tensor for the corresponding Lie algebra, $\boldsymbol{F}=\mathrm{d} \boldsymbol{A}+\boldsymbol{A} \boldsymbol{A}$ is the corresponding two-form curvature and $k$ is a constant [4].

Using theorem VII.2 of Ref. [2], it is possible to show that the only non-vanishing components of an invariant tensor for the $\mathfrak{B}$ algebra are given by

$$
\begin{aligned}
\left\langle\boldsymbol{J}_{a_{1} a_{2}} \boldsymbol{J}_{a_{3} a_{4}} \boldsymbol{P}_{a_{5}}\right\rangle & =\alpha_{1} \frac{4 l^{3}}{3} \epsilon_{a_{1} \cdots a_{5}}, \\
\left\langle\boldsymbol{J}_{a_{1} a_{2}} \boldsymbol{J}_{a_{3} a_{4}} \boldsymbol{Z}_{a_{5}}\right\rangle & =\alpha_{3} \frac{4 l^{3}}{3} \epsilon_{a_{1} \cdots a_{5}}, \\
\left\langle\boldsymbol{J}_{a_{1} a_{2}} \boldsymbol{Z}_{a_{3} a_{4}} \boldsymbol{P}_{a_{5}}\right\rangle & =\alpha_{3} \frac{4 l^{3}}{3} \epsilon_{a_{1} \cdots a_{5}},
\end{aligned}
$$

where $\alpha_{1}$ and $\alpha_{3}$ are arbitrary independent constants of dimensions $[\text { length }]^{-3}$.

Using the extended Cartan homotopy formula as in Ref. [5], and integrating by parts, it is possible to write down the Chern-Simons Lagrangian in five dimensions for the $\mathcal{B}$ algebra as

$$
\begin{aligned}
& L_{\mathrm{EChS}}^{(5)}=\alpha_{1} l^{2} \epsilon_{a b c d e} e^{a} R^{b c} R^{d e} \\
& \quad+\alpha_{3} \epsilon_{a b c d e}\left(\frac{2}{3} R^{a b} e^{c} e^{d} e^{e}+2 l^{2} k^{a b} R^{c d} T^{e}+l^{2} R^{a b} R^{c d} h^{e}\right) \\
& \quad+\mathrm{d} B_{\mathrm{EChS}}^{(4)}
\end{aligned}
$$

where the surface term $B_{\mathrm{EChS}}^{(4)}$ is given by

$$
\begin{aligned}
& B_{\mathrm{EChS}}^{(4)}=\alpha_{1} l^{2} \epsilon_{a b c d e} e^{a} \omega^{b c}\left(\frac{2}{3} \mathrm{~d} \omega^{d e}+\frac{1}{2} \omega^{d}{ }_{f} \omega^{f e}\right) \\
& +\alpha_{3} \epsilon_{a b c d e}\left[l^{2}\left(h^{a} \omega^{b c}+k^{a b} e^{c}\right)\left(\frac{2}{3} \mathrm{~d} \omega^{d e}+\frac{1}{2} \omega^{d}{ }_{f} \omega^{f e}\right)\right. \\
& \left.+l^{2} k^{a b} \omega^{c d}\left(\frac{2}{3} \mathrm{~d} e^{e}+\frac{1}{2} \omega^{d}{ }_{f} e^{e}\right)+\frac{1}{6} e^{a} e^{b} e^{c} \omega^{d e}\right]
\end{aligned}
$$

and where $\alpha_{1}, \alpha_{3}$ are parameters of the theory, $l$ is a coupling constant, $R^{a b}=\mathrm{d} \omega^{a b}+\omega^{a}{ }_{c} \omega^{c b}$ corresponds to the curvature two-form in the first-order formalism related to the one-form spin connection [4,6,7], and $e^{a}, h^{a}$, and $k^{a b}$ are the other gauge fields present in the theory [1].

From (5) we can see that the third term is a surface term and can be removed from this Lagrangian. Thus

$$
\begin{aligned}
& L_{\mathrm{EChS}}^{(5)}=\alpha_{1} l^{2} \varepsilon_{a b c d e} R^{a b} R^{c d} e^{e} \\
& \quad+\alpha_{3} \epsilon_{a b c d e}\left(\frac{2}{3} R^{a b} e^{c} e^{d} e^{e}+2 l^{2} k^{a b} R^{c d} T^{e}+l^{2} R^{a b} R^{c d} h^{e}\right)
\end{aligned}
$$

is the Einstein-Chern-Simons Lagrangian studied in Ref. [1].

The absence should be noted of kinetic terms for the fields $h^{a}$ and $k^{a b}$ in (7). The kinetic terms for the $h^{a}$ and $k^{a b}$ fields are present in the surface term of the Lagrangian (5) given by (6).

The Lagrangian (7) shows that standard, five-dimensional General Relativity emerges as the $l \rightarrow 0$ limit of a CS theory for the generalized Poincaré algebra $\mathfrak{B}$. Here $l$ is a length scale, a coupling constant that characterizes different regimes within the theory. The $\mathfrak{B}$ algebra, on the other hand, is constructed from the AdS algebra and a particular semigroup $S$ by means of the S-expansion procedure. The field content induced by the $\mathfrak{B}$ algebra includes the vielbein $e^{a}$, the spin connection $\omega^{a b}$, and two extra bosonic fields $h^{a}$ and $k^{a b}$, which can be interpreted as boson fields coupled to the field curvature and the parameter $l^{2}$ can be interpreted as a kind of coupling constant.

Recently was found [8] that the standard five-dimensional FRW equations and some of their solutions can be obtained, in a certain limit, from the so-called Chern-Simons-FRW field equations, which are the cosmological field equations corresponding to a Chern-Simons gravity theory.

It is the purpose of this paper to show that the EinsteinChern-Simons (EChS) field equations, subject to (i) the torsion-free condition $\left(T^{a}=0\right)$, and (ii) the variation of the matter Lagrangian with respect to (w.r.t.) the spin connection is zero $\left(\delta L_{M} / \delta \omega^{a b}=0\right)$, can be written in a similar way to the Einstein-Maxwell field equations. The interpretation of the $h^{a}$ field as a perfect fluid allow us to show that the Einstein-Chern-Simons field equations have a universe in accelerated expansion as one of their solutions.

This paper is organized as follows: In Sect. 2 we briefly review the Einstein-Chern-Simons field equations. In Sect. 3 we study the Einstein-Chern-Simons field equations in the range of validity of General Relativity. In Sect. 5 we consider accelerated solutions for the Einstein-Chern-Simons field equations. We try to find solutions that describe accelerated expansion for the cases of open universes, flat universes, and closed universes. In Sect. 6 we consider the consistency of the solutions with the "era of matter". A summary and an appendix conclude this work.

\section{Einstein-Chern-Simons field equations}

In Ref. [8] it was found that in the presence of matter the Lagrangian is given by

$$
L=L_{\mathrm{ChS}}^{(5)}+\kappa L_{M}
$$

where $L_{\mathrm{ChS}}^{(5)}$ is the five-dimensional Chern-Simons Lagrangian given by (7), $L_{M}=L_{M}\left(e^{a}, h^{a}, \omega^{a b}\right)$ is the matter 
Lagrangian, and $\kappa$ is a coupling constant related to the effective Newton constant. The variation of the Lagrangian (8) w.r.t. the dynamical fields vielbein $e^{a}$, spin connection $\omega^{a b}$, $h^{a}$, and $k^{a b}$, leads to the following field equations:

$$
\begin{aligned}
& \varepsilon_{a b c d e}\left(2 \alpha_{3} R^{a b} e^{c} e^{d}+\alpha_{1} l^{2} R^{a b} R^{c d}\right. \\
& \left.+2 \alpha_{3} l^{2} D_{\omega} k^{a b} R^{c d}\right)=\kappa \frac{\delta L_{M}}{\delta e^{e}}, \\
& \alpha_{3} l^{2} \varepsilon_{a b c d e} R^{a b} R^{c d}=\kappa \frac{\delta L_{M}}{\delta h^{e}}, \\
& 2 \alpha_{3} l^{2} \varepsilon_{a b c d e} R^{c d} T^{e}=\kappa \frac{\delta L_{M}}{\delta k^{a b}}, \\
& 2 \varepsilon_{a b c d e}\left(\alpha_{1} l^{2} R^{c d} T^{e}+\alpha_{3} l^{2} D_{\omega} k^{a b} T^{e}\right. \\
& \left.+\alpha_{3} e^{c} e^{d} T^{e}+\alpha_{3} l^{2} R^{c d} D_{\omega} h^{e}\right) \\
& +2 \alpha_{3} \varepsilon_{a b c d e} l^{2} R^{c d} k_{f}^{e} e^{f}=\kappa \frac{\delta L_{M}}{\delta \omega^{a b}} .
\end{aligned}
$$

For simplicity, we will assume that the torsion vanishes $\left(T^{a}=0\right)$ and $k^{a b}=0$. In this case (9)-(12) take the form

$\varepsilon_{a b c d e}\left(2 \alpha_{3} R^{a b} e^{c} e^{d}+\alpha_{1} l^{2} R^{a b} R^{c d}\right)=\kappa \frac{\delta L_{M}}{\delta e^{e}}$,

$\alpha_{3} l^{2} \varepsilon_{a b c d e} R^{a b} R^{c d}=\kappa \frac{\delta L_{M}}{\delta h^{e}}$,

$\frac{\delta L_{M}}{\delta k^{a b}}=0$

$2 \alpha_{3} l^{2} \varepsilon_{a b c d e} R^{c d} D_{\omega} h^{e}=\kappa \frac{\delta L_{M}}{\delta \omega^{a b}}$.

This field equations system can be written in the form

$$
\begin{aligned}
& \varepsilon_{a b c d e} R^{a b} e^{c} e^{d}=4 \kappa_{5}\left(\frac{\delta L_{M}}{\delta e^{e}}+\alpha \frac{\delta L_{M}}{\delta h^{e}}\right), \\
& l^{2} \varepsilon_{a b c d e} R^{a b} R^{c d}=8 \kappa_{5} \frac{\delta L_{M}}{\delta h^{e}}, \\
& l^{2} \varepsilon_{a b c d e} R^{c d} D_{\omega} h^{e}=4 \kappa_{5} \frac{\delta L_{M}}{\delta \omega^{a b}}
\end{aligned}
$$

where we introduce $\kappa_{5}=\kappa / 8 \alpha_{3}$ and $\alpha=-\alpha_{1} / \alpha_{3}$.

The field equation (9) contains three terms. The first one is proportional to the Einstein tensor. The second one corresponds to a quadratic term in the curvature, and a third one, a term that describes the dynamics of the field $k^{a b}$. Since we assume $k^{a b}=0$ the last term in left side of (9) vanishes.

In order to write this field equation manner analogous to Einstein's equations, one chooses to leave the term proportional to the Einstein tensor on the left side of (9),

$\epsilon_{a b c d e} R^{b c} e^{d} e^{e}=\frac{\kappa}{2 \alpha_{3}} \frac{\delta L_{M}}{\delta e^{a}}-\frac{\alpha_{1}}{2 \alpha_{3}} l^{2} \epsilon_{a b c d e} R^{b c} R^{d e}$

and using (14) we obtain (17).

This result allows us to interpret $\delta L_{M} / \delta h^{a}$ as the energymomentum tensor for a second type of matter, not ordinary.
Henceforth we will say that $\delta L_{M} / \delta h^{a}$ corresponds to the energy-momentum tensor for the field $h^{a}$.

The equation of motion for the $h^{a}$-field is given by (19). The condition $\delta L_{M} / \delta \omega^{a b}=0$ (usual in gravity theories), imposed for consistency with the condition $T^{a}=0$, leads to the equation of motion (22) for the $h^{a}$-field. This means that $h^{a}$-field is governed by the following field equations:

$\varepsilon_{a b c d e} R^{a b} e^{c} e^{d}=4 \kappa_{5}\left(\frac{\delta L_{M}}{\delta e^{e}}+\alpha \frac{\delta L_{M}}{\delta h^{e}}\right)$,

$\frac{l^{2}}{8 \kappa_{5}} \varepsilon_{a b c d e} R^{a b} R^{c d}=\frac{\delta L_{M}}{\delta h^{e}}$,

$\varepsilon_{\text {abcde }} R^{c d} D_{\omega} h^{e}=0$.

This means that the Einstein-Chern-Simons field equations, subject to the conditions $T^{a}=0, k^{a b}=0$, and $\delta L_{M} / \delta \omega^{a b}=0$, can be rewritten in a way similar to the Einstein-Maxwell field equations.

From (20) to (22) we can see that if $L_{M}=0$, then in five dimensions there is no solution of Schwarzschild type [1,9].

\section{Einstein-Chern-Simons equations in the range of validity of general relativity}

From (20)-(21) we can see that General Relativity is valid when (i) the curvature $R^{a b}$ takes values not excessively large (ii) the parameter $l$ takes small values $(l \longrightarrow 0)$ [1]; (iii) the constant $\alpha$ takes values that are not excessively large. In fact, in this case we see that (21) takes the form

$\frac{\delta L_{M}}{\delta h^{e}} \approx 0$.

Introducing (23) into (20) we obtain the Einstein field equation

$\varepsilon_{\text {abcde }} R^{a b} e^{c} e^{d} \approx 4 \kappa_{5} \frac{\delta L_{M}}{\delta e^{e}}$.

If $R^{a b}$ is not large then $\delta L_{M} / \delta e^{a}$ is also not large. This means that General Relativity can be seen as a low energy limit of Einstein-Chern-Simons gravity. Thus, in the range of validity of General Relativity, (20)-(22) are given by

$\varepsilon_{a b c d e} R^{a b} e^{c} e^{d}=4 \kappa_{5} \frac{\delta L_{M}}{\delta e^{e}}$,

$\varepsilon_{a b c d e} R^{c d} D_{\omega} h^{e}=0$.

On the another hand, if $R^{a b}$ is large enough, so that when it is multiplied by $l^{2}$ (which is very small) it will have nonnegligible results, then we will find that $\delta L_{M} / \delta h^{a}$ is not negligible. This means that, in this case, we must consider the entire system of (20)-(22). 


\section{Einstein-Chern-Simons field equations for a Friedmann-Robertson-Walker-like spacetime}

The shape of the field $e^{a}$ is obtained from of the application of the cosmological principle to the metric tensor of spacetime: it is considered a splitting of the 5D-manifold in a maximally symmetric four-dimensional manifold and one temporal dimension ( $M=R \times \Sigma_{4}$ ). This leads to a fivedimensional Friedmann-Robertson-Walker (FRW) metric. Thus the vielbein can be chosen like in [8]:

$e^{0}=\mathrm{d} t$,

$e^{1}=\frac{a(t)}{\sqrt{1-k r^{2}}} \mathrm{~d} r$

$e^{2}=a(t) r \mathrm{~d} \theta_{2}$,

$e^{3}=a(t) r \sin \theta_{2} \mathrm{~d} \theta_{3}$,

$e^{4}=a(t) r \sin \theta_{2} \sin \theta_{3} \mathrm{~d} \theta_{4}$

where $a(t)$ is the scale factor of the universe and $k$ is the sign of the curvature of space $\left(\Sigma_{4}\right)$ : (i) +1 for a closed space $\left(S^{4}\right)$, (ii) 0 for a flat space $\left(E^{4}\right)$, and (iii) -1 for an open space (hyperbolic).

The application of the cosmological principle to the metric tensor of the spacetime also constrains the shape of the field $h^{a}$ (see for example [8]). A detailed discussion can be also found in Ref. [10]. The bosonic field $h^{a}$ is given by

$h^{0}=h(0) \mathrm{d} t=h(0) e^{0}$,

$h^{1}=h(t) \frac{a(t)}{\sqrt{1-k r^{2}}} \mathrm{~d} r=h(t) e^{1}$,

$h^{2}=h(t) a(t) r \mathrm{~d} \theta_{2}=h(t) e^{2}$,

$h^{3}=h(t) a(t) r \sin \theta_{2} \mathrm{~d} \theta_{3}=h(t) e^{3}$,

$h^{4}=h(t) a(t) r \sin \theta_{2} \sin \theta_{3} \mathrm{~d} \theta_{4}=h(t) e^{4}$,

where $h(0)$ is a constant and $h(t)$ is a function of time $t$, which must be determined. Substituting (28) into (22) we obtain the explicit form of the equations of motion for the $h^{a}$-field, which will be displayed in (39).

In accordance with (20), we will consider a fluid composed of two perfect fluids, the first one related to ordinary energymomentum tensor $\left(T_{\mu \nu} \sim \frac{\delta L_{M}}{\delta e^{a}}\right)$ and the second one related to the field $h^{a}\left(T_{\mu \nu}^{(h)} \sim \frac{\delta L_{M}}{\delta h^{a}}\right)$. The energy-momentum tensors in the comoving frame are given by

$T_{\mu \nu}=\operatorname{diag}(\rho, p, p, p, p)$,

$T_{\mu \nu}^{(h)}=\operatorname{diag}\left(\rho^{(h)}, p^{(h)}, p^{(h)}, p^{(h)}, p^{(h)}\right)$,

where $\rho$ is the matter density and $p$ is the pressure of fluid. Then the energy-momentum tensor for the composed fluid is

$\tilde{T}_{\mu \nu}=T_{\mu \nu}+\alpha T_{\mu \nu}^{(h)}$

$$
\begin{aligned}
= & \operatorname{diag}\left(\rho+\alpha \rho^{(h)}, p+\alpha p^{(h)}\right. \\
& \left.p+\alpha p^{(h)}, p+\alpha p^{(h)}, p+\alpha p^{(h)}\right) \\
= & \operatorname{diag}(\tilde{\rho}, \tilde{p}, \tilde{p}, \tilde{p}, \tilde{p}) .
\end{aligned}
$$

In the torsion-free case, the energy-momentum tensor of ordinary matter satisfies a conservation equation and the Einstein tensor has also a zero divergence. In this case the energy-momentum tensor for the non-ordinary matter must also satisfy a conservation equation. In fact, from (20) we find

$\nabla_{\mu} T_{\nu}^{\mu}=0, \quad \nabla_{\mu} T_{\nu}^{(h) \mu}=0$

Introducing (27)-(33) into (20)-(22) we find the following field equations (see Ref. [8] and Appendix A):

$6\left(\frac{\dot{a}^{2}+k}{a^{2}}\right)=\kappa_{5} \tilde{\rho}$,

$3\left[\frac{\ddot{a}}{a}+\left(\frac{\dot{a}^{2}+k}{a^{2}}\right)\right]=-\kappa_{5} \tilde{p}$,

$\frac{3 l^{2}}{\kappa_{5}}\left(\frac{\dot{a}^{2}+k}{a^{2}}\right)^{2}=\rho^{(h)}$,

$\frac{3 l^{2}}{\kappa_{5}} \frac{\ddot{a}}{a}\left(\frac{\dot{a}^{2}+k}{a^{2}}\right)=-p^{(h)}$,

$\left(\frac{\dot{a}^{2}+k}{a^{2}}\right)\left[(h-h(0)) \frac{\dot{a}}{a}+\dot{h}\right]=0$.

We should note that (35) was studied in Ref. [11] in the context of inflationary cosmology.

Equations (35) and (36) are very similar to the Friedmann equations in five dimensions. However, now $\rho$ and $p$ are subject to restrictions imposed by the remaining equations.

\section{Accelerated solution for Einstein-Chern-Simons field equations}

In order to recover the known results of the standard cosmology in the context of accelerated expansion we use the approach

$T_{\mu \nu} \ll \alpha T_{\mu \nu}^{(h)}$.

This approach is analogous to the case when, in the era of dark energy, the energy-momentum tensor is neglected compared to the cosmological constant. This means that the contribution from the ordinary matter is negligible compared to the contribution from the field $h^{a}$. In this case, the energymomentum tensor $\tilde{T}_{\mu \nu}$ of the fluid is given by

$$
\begin{aligned}
\tilde{T}_{\mu \nu} & =\operatorname{diag}(\tilde{\rho}, \tilde{p}, \tilde{p}, \tilde{p}, \tilde{p}) \\
& =\alpha T_{\mu \nu}^{(h)} \\
& =\operatorname{diag}\left(\alpha \rho^{(h)}, \alpha p^{(h)}, \alpha p^{(h)}, \alpha p^{(h)}, \alpha p^{(h)}\right)
\end{aligned}
$$


and (35)-(39) take the form

$6\left(\frac{\dot{a}^{2}+k}{a^{2}}\right)=\kappa_{5} \alpha \rho^{(h)}$,

$3\left[\frac{\ddot{a}}{a}+\left(\frac{\dot{a}^{2}+k}{a^{2}}\right)\right]=-\kappa_{5} \alpha p^{(h)}$,

$\frac{3 l^{2}}{\kappa_{5}}\left(\frac{\dot{a}^{2}+k}{a^{2}}\right)^{2}=\rho^{(h)}$,

$\frac{3 l^{2}}{\kappa_{5}} \frac{\ddot{a}}{a}\left(\frac{\dot{a}^{2}+k}{a^{2}}\right)=-p^{(h)}$,

$\left(\frac{\dot{a}^{2}+k}{a^{2}}\right)\left[(h-h(0)) \frac{\dot{a}}{a}+\dot{h}\right]=0$.

5.1 Case $T_{\mu \nu}=0$ and $k=-1$

Introducing (43) into (41) we obtain

$6\left(\frac{\dot{a}^{2}+k}{a^{2}}\right)=3 l^{2} \alpha\left(\frac{\dot{a}^{2}+k}{a^{2}}\right)^{2}$,

which can be rewritten

$$
\left(\frac{\dot{a}^{2}+k}{a^{2}}\right)\left(\frac{2}{\alpha l^{2}}-\frac{\dot{a}^{2}+k}{a^{2}}\right)=0 .
$$

\section{Solution $\ddot{a}=0$}

Consider the solution $\ddot{a}=0$, i.e., a solution without accelerated expansion. For the first term in the left side of (47) we have

$\frac{\dot{a}^{2}+k}{a^{2}}=0$,

remembering $k=-1$, we have

$\dot{a}=\sqrt{-k}$.

The solution is

$a(t)=\sqrt{-k}\left(t-t_{0}\right)+a_{0}$.

In this case $a(t)$ increases linearly, i.e., there is no accelerated expansion.

Substituting this solution into (41)-(44) we find

$\rho^{(h)}=p^{(h)}=0$,

and (45) is satisfied for $h(t)$ arbitrary.

2. Solution $\ddot{a} \neq 0$

From (47) we obtain

$\dot{a}^{2}-\frac{2}{\alpha l^{2}} a^{2}=-k$.

From (52) we can see two options: (i) $\alpha>0$ and (ii) $\alpha<0$. a. Case $\alpha>0$.

Consider the case where the constant $\alpha$ is positive. Using the following ansatz ${ }^{1}$

$a(t)=A \sin h\left(\sqrt{\frac{2}{\alpha l^{2}}}\left(t-t^{\prime}\right)\right)$

where $t^{\prime}$ is a constant of integration, we obtain

$A=\sqrt{-\frac{\alpha l^{2} k}{2}}$

and therefore

$a(t)=\sqrt{-\frac{\alpha l^{2} k}{2}} \sin h\left(\sqrt{\frac{2}{\alpha l^{2}}}\left(t-t^{\prime}\right)\right)$,

the initial condition $a_{0}=a\left(t=t_{0}\right)$ leads to

$$
\begin{aligned}
a(t)= & \sqrt{-\frac{\alpha l^{2} k}{2}} \\
& \times \sin h\left[\sqrt{\frac{2}{\alpha l^{2}}}\left(t-t_{0}\right)+\operatorname{arsinh}\left(\sqrt{-\frac{2}{\alpha l^{2} k}} a_{0}\right)\right]
\end{aligned}
$$

and

$$
\begin{aligned}
\dot{a}(t)= & \sqrt{-k} \\
& \times \cos h\left[\sqrt{\frac{2}{\alpha l^{2}}}\left(t-t_{0}\right)+\operatorname{arsinh}\left(\sqrt{-\frac{2}{\alpha l^{2} k}} a_{0}\right)\right] .
\end{aligned}
$$

This result shows that if $\alpha>0$, then there is an accelerated expansion (see Fig. 1).

On the another hand, from (56) and (57) we can see that

$\ddot{a}(t)=\frac{2}{\alpha l^{2}} a(t)$,

substituting (56), (57), and (58) into (41)-(44) we obtain

$\rho^{(h)}=-p^{(h)}=\frac{12}{\kappa_{5} \alpha l^{2}}$,

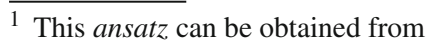

$\dot{a}=\sqrt{\frac{2}{\alpha l^{2}} a^{2}-k}$,

whose solution is $(\alpha>0, k=-1)$

$\int_{t^{\prime}}^{t} \frac{\mathrm{d} a}{\sqrt{\frac{2}{\alpha l^{2}} a^{2}-k}}=t-t^{\prime}$

using a hyperbolic substitution

$\sqrt{\frac{\alpha l^{2}}{2}} \operatorname{arsinh}\left(\sqrt{-\frac{2}{\alpha l^{2} k}} a\right)=t-t^{\prime}$. 


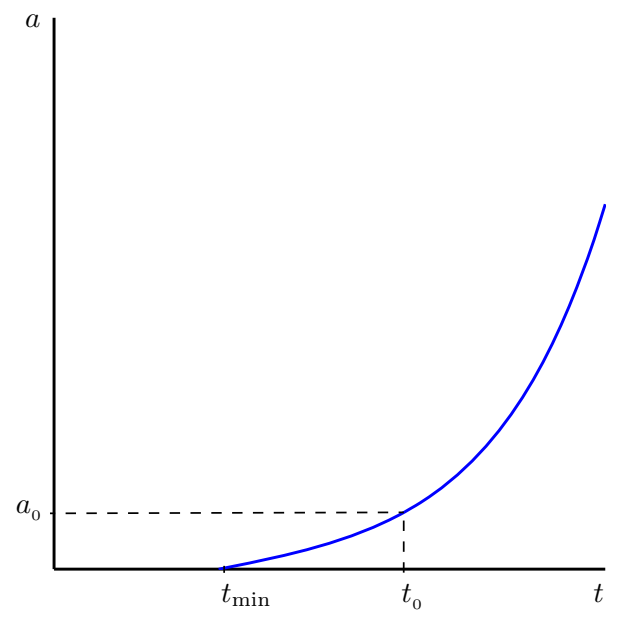

Fig. 1 Graph of $a(t)$ with $\alpha>0$ and $k=-1$. See (56)

i.e., we have an accelerated expansion when the energy density is positive and pressure is negative (like a positive cosmological constant).

From (45) we find

$-\frac{\dot{h}}{h-h(0)}=\frac{\dot{a}}{a}$.

Integrating, we find

$h(t)=\frac{C}{\sin h\left[\sqrt{\frac{2}{\alpha l^{2}}}\left(t-t_{0}\right)+\operatorname{arsinh}\left(\sqrt{-\frac{2}{\alpha l^{2} k}} a_{0}\right)\right]}+h(0)$

where $C$ is a constant of integration. The initial condition $h_{0}=h\left(t_{0}\right)$ leads to

$h(t)=\frac{\left(h_{0}-h(0)\right) \sqrt{\frac{2}{\alpha l^{2} k}} a_{0}}{\sin h\left[\sqrt{\frac{2}{\alpha l^{2}}}\left(t-t_{0}\right)+\operatorname{arsinh}\left(\sqrt{-\frac{2}{\alpha l^{2} k}} a_{0}\right)\right]}+h(0)$

from which we can see that $h(t) \rightarrow h(0)$ when $t \rightarrow \infty$

b. Case $\alpha<0$.

Consider now the case when the constant $\alpha$ is negative. The ansatz.

$a(t)=A \sin \left(\sqrt{-\frac{2}{\alpha l^{2}}}\left(t-t^{\prime}\right)\right)$,

with $t^{\prime}$ a constant of integration, leads to

$A=\sqrt{\frac{\alpha l^{2} k}{2}}$

therefore

$a(t)=\sqrt{\frac{\alpha l^{2} k}{2}} \sin \left(\sqrt{-\frac{2}{\alpha l^{2}}}\left(t-t^{\prime}\right)\right)$.

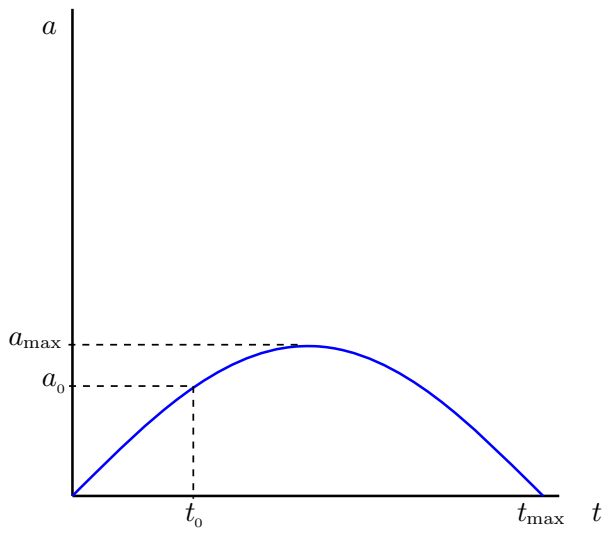

Fig. 2 Graph of $a(t)$ with $\alpha<0$ and $k=-1$. See (65)

The initial condition $a_{0}=a\left(t=t_{0}\right)$ leads to

$$
\begin{aligned}
a(t)= & \sqrt{\frac{\alpha l^{2} k}{2}} \\
& \times \sin \left[\sqrt{-\frac{2}{\alpha l^{2}}}\left(t-t_{0}\right)+\arcsin \left(\sqrt{\frac{2}{\alpha l^{2} k}} a_{0}\right)\right]
\end{aligned}
$$

and

$$
\begin{aligned}
\dot{a}(t)= & \sqrt{-k} \\
& \times \cos \left[\sqrt{-\frac{2}{\alpha l^{2}}}\left(t-t_{0}\right)+\arcsin \left(\sqrt{\frac{2}{\alpha l^{2} k}} a_{0}\right)\right] .
\end{aligned}
$$

Therefore if $a(t)>0$ then $\ddot{a}(t)<0$, which shows that if $\alpha<0$, then there is a decelerated expansion (see Fig. 2).

On the another hand, substituting (65) and (66) into (41) (44) we obtain

$\rho^{(h)}=-p^{(h)}=\frac{12}{\kappa_{5} \alpha l^{2}}$.

Since the energy-momentum tensor is given by

$$
\tilde{T}_{\mu \nu}=\alpha T_{\mu \nu}^{(h)}=\operatorname{diag}\left(\alpha \rho^{(h)}, \alpha p^{(h)}, \alpha p^{(h)}, \alpha p^{(h)}, \alpha p^{(h)}\right),
$$

we find that the corresponding energy density and pressure are $(\alpha<0)$

$\tilde{\rho}=\alpha \rho^{(h)}=\frac{12}{\kappa_{5} \alpha l^{2}}<0$,
$\tilde{p}=\alpha p^{(h)}=-\frac{12}{\kappa_{5} \alpha l^{2}}>0$,

i.e., the energy density is negative and the pressure is positive (like a negative cosmological constant). 
From (45) we find

$-\frac{\dot{h}}{h-h(0)}=\frac{\dot{a}}{a}$.

Integrating, we find

$h(t)=\frac{C}{\sin \left[\sqrt{-\frac{2}{\alpha l^{2}}}\left(t-t_{0}\right)+\arcsin \left(\sqrt{\frac{2}{\alpha l^{2} k}} a_{0}\right)\right]}+h(0)$

where $C$ is a constant of integration. The initial condition $h_{0}=h\left(t_{0}\right)$ leads to

$h(t)=\frac{\left(h_{0}-h(0)\right) \sqrt{\frac{2}{\alpha l^{2} k}} a_{0}}{\sin \left[\sqrt{-\frac{2}{\alpha l^{2}}}\left(t-t_{0}\right)+\arcsin \left(\sqrt{\frac{2}{\alpha l^{2} k}} a_{0}\right)\right]}+h(0)$.

5.2 Case $T_{\mu \nu}=0$ and $k=0$

Introducing (43) into (41) and considering $k=0$, we obtain

$6\left(\frac{\dot{a}}{a}\right)^{2}=3 l^{2} \alpha\left(\frac{\dot{a}}{a}\right)^{4}$,

which can be rewritten as

$\left(\frac{\dot{a}}{a}\right)^{2}\left(\frac{2}{\alpha l^{2}}-\frac{\dot{a}^{2}}{a^{2}}\right)=0$.

1. Static solution $\dot{a}=0$

The solution for an static universe is given by

$a(t)=a_{0}$,

which leads to

$\rho^{(h)}=p^{(h)}=0$

and (45) is satisfied for all $h(t)$.

\section{Non-static solution $\dot{a} \neq 0$}

From (75) we obtain

$\dot{a}^{2}-\frac{2}{\alpha l^{2}} a^{2}=0$

This equation has a solution, only if $\alpha>0$.

a. Case $\alpha>0$.

In this case we have an expanding universe,

$a(t)=A \exp \left(\sqrt{\frac{2}{\alpha l^{2}}} t\right)$.
The initial condition $a_{0}=a\left(t_{0}\right)$ leads to

$a(t)=a_{0} \exp \left(\sqrt{\frac{2}{\alpha l^{2}}}\left(t-t_{0}\right)\right)$

and

$\rho^{(h)}=-p^{(h)}=\frac{12}{\kappa_{5} \alpha l^{2}}$.

Substituting (80) into (45), solving for $h(t)$ and using the initial condition $h_{0}=h\left(t_{0}\right)$, we find

$h(t)=\frac{h_{0}-h(0)}{\exp \left(\sqrt{\frac{2}{\alpha l^{2}}}\left(t-t_{0}\right)\right)}+h(0)$.

b. Case $\alpha<0$.

In this case it is not possible to find a solution.

5.3 Case $T_{\mu \nu}=0$ and $k=1$

Introducing (43) into (41) we obtain

$6\left(\frac{\dot{a}^{2}+k}{a^{2}}\right)=3 l^{2} \alpha\left(\frac{\dot{a}^{2}+k}{a^{2}}\right)^{2}$,

which can be rewritten as

$\left(\frac{\dot{a}^{2}+k}{a^{2}}\right)\left(\frac{2}{\alpha l^{2}}-\frac{\dot{a}^{2}+k}{a^{2}}\right)=0$.

1. Case $\ddot{a}=0$.

In this case it is not possible to find a solution.

2. Case $\ddot{a} \neq 0$.

From (83) we obtain

$\frac{2}{\alpha l^{2}} a^{2}-\dot{a}^{2}=k$.

From (84) we can see two cases.

a. Case $\alpha>0$.

If $\alpha>0$ we can postulate a solution given by

$a(t)=A \cos h\left(\sqrt{\frac{2}{\alpha l^{2}}}\left(t-t^{\prime}\right)\right)$

where $t^{\prime}$ is a constant of integration, which leads to

$A=\sqrt{\frac{\alpha l^{2} k}{2}}$. 


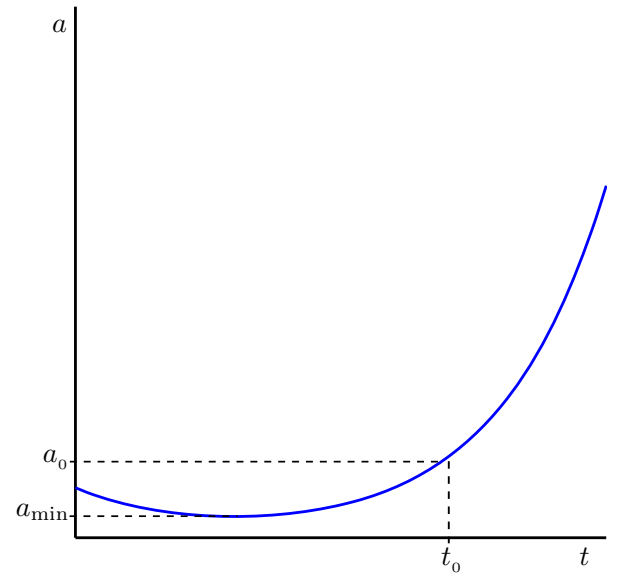

Fig. 3 Graph of $a(t)$ with $\alpha>0$ and $k=1$. See (87)

The initial condition $a_{0}=a\left(t=t_{0}\right)$ leads to

$a(t)=\sqrt{\frac{\alpha l^{2} k}{2}} \cos h\left[\sqrt{\frac{2}{\alpha l^{2}}}\left(t-t^{\prime}\right)+\operatorname{arcos} h\left(\sqrt{\frac{2}{\alpha l^{2}}} a_{0}\right)\right]$

and

$\dot{a}(t)=\sqrt{k} \sin h\left[\sqrt{\frac{2}{\alpha l^{2}}}\left(t-t_{0}\right)+\operatorname{arcos} h\left(\sqrt{\frac{2}{\alpha l^{2}}} a_{0}\right)\right]$,

which shows an accelerated expansion (see Fig. 3).

Substituting (87) and (88) into (41)-(44) we obtain

$\rho^{(h)}=-p^{(h)}=\frac{12}{\kappa_{5} \alpha l^{2}}$,

i.e., we have an accelerated expansion when the energy density is positive and the pressure is negative (like a positive cosmological constant).

From (45) we find

$-\frac{\dot{h}}{h-h(0)}=\frac{\dot{a}}{a}$,

so that

$h(t)=\frac{C}{\cos h\left[\sqrt{\frac{2}{\alpha l^{2}}}\left(t-t_{0}\right)+\operatorname{arcos} h\left(\sqrt{\frac{2}{\alpha l^{2} k}} a_{0}\right)\right]}+h(0)$

where $C$ is a constant of integration. The initial condition $h_{0}=h\left(t_{0}\right)$ leads to

$h(t)=\frac{\left(h_{0}-h(0)\right) \sqrt{\frac{2}{\alpha l^{2} k}} a_{0}}{\cos h\left[\sqrt{\frac{2}{\alpha l^{2}}}\left(t-t_{0}\right)+\operatorname{arcos} h\left(\sqrt{\frac{2}{\alpha l^{2} k}} a_{0}\right)\right]}+h(0)$,

from which we can see that $h(t) \rightarrow h(0)$ when $t \rightarrow \infty$.
Table 1 Solutions for scale factor of an open space $k=-1$ (hyperbolic)

\begin{tabular}{lllll}
\hline $\begin{array}{l}\text { Dynamics } \\
a(t)\end{array}$ & $\alpha$ & $\rho^{(h)}$ & $p^{(h)}$ & $\begin{array}{l}\Lambda \\
\text { compatible }\end{array}$ \\
\hline Accelerated & $>0$ & $>0$ & $<0$ & $>0$ \\
$\begin{array}{l}\text { Decelerated } \\
\text { No accelerated } \\
\text { (vacuum) }\end{array}$ & $<0$ & $<0$ & $>0$ & $<0$ \\
\hline
\end{tabular}

Table 2 Solutions for scale factor of a flat space, $k=0$

\begin{tabular}{|c|c|c|c|c|}
\hline $\begin{array}{l}\text { Dynamics } \\
a(t)\end{array}$ & $\alpha$ & $\rho^{(h)}$ & $p^{(h)}$ & $\begin{array}{l}\Lambda \\
\text { compatible }\end{array}$ \\
\hline Accelerated & $>0$ & $>0$ & $<0$ & $>0$ \\
\hline $\begin{array}{l}\text { Stationary } \\
\text { (vacuum) }\end{array}$ & Any & 0 & 0 & - \\
\hline
\end{tabular}

Table 3 Solutions for scale factor of a closed space, $k=1$

\begin{tabular}{lllll}
\hline $\begin{array}{l}\text { Dynamics } \\
a(t)\end{array}$ & $\alpha$ & $\rho^{(h)}$ & $p^{(h)}$ & $\begin{array}{l}\Lambda \\
\text { compatible }\end{array}$ \\
\hline Accelerated & $>0$ & $>0$ & $<0$ & $>0$ \\
\hline
\end{tabular}

b. Case $\alpha<0$.

If $\alpha<0$ (84) has no solution.

\subsection{Era of dark energy from Einstein-Chern-Simons gravity}

The results in the previous section are summarized in Tables 1, 2, and 3 .

Thus we have found solutions that describe accelerated expansion for the three possible cosmological models of the universe. Namely, spherical expansion $(k=1)$, flat expansion $(k=0)$, and hyperbolic expansion $(k=-1)$ when the constant $\alpha$ is greater than zero. This means that the EinsteinChern-Simons field equations have as one of their solutions a universe in accelerated expansion. This result allows us to conjecture that these solutions are compatible with the era of dark energy and that the energy-momentum tensor for the field $h^{a}$ corresponds to a form of positive cosmological constant.

From these solutions we can see that as time passes, the $h(t)$ decreases rapidly to $h(0)$, a constant value, keeping a constant matter density.

We have also shown that the EChS field equations have solutions that allow us to identify the energy-momentum tensor for the field $h^{a}$ with a negative cosmological constant. 


\section{Consistency of the solutions with the "era of matter"}

In the previous section, we find that the solutions of the EChS field equations, with $T_{\mu \nu}=0$, can be useful as models of the era of dark energy. In this section we review the consistency of these equations with the era of matter.

We will consider the ordinary matter as dust $(\rho \neq 0$, $p=0$ ), such as occurs in standard cosmology. The nonordinary matter will be modeled as a perfect fluid $\left(\rho^{(h)} \neq 0\right.$ y $p^{(h)} \neq 0$ ). In this case the field equations (35)-(39) take the form

$$
\begin{aligned}
& 6\left(\frac{\dot{a}^{2}+k}{a^{2}}\right)=\kappa_{5}\left(\rho+\alpha \rho^{(h)}\right) \\
& 3\left[\frac{\ddot{a}}{a}+\left(\frac{\dot{a}^{2}+k}{a^{2}}\right)\right]=-\kappa_{5} \alpha p^{(h)} \\
& \frac{3 l^{2}}{\kappa_{5}}\left(\frac{\dot{a}^{2}+k}{a^{2}}\right)^{2}=\rho^{(h)} \\
& \frac{3 l^{2}}{\kappa_{5}} \frac{\ddot{a}}{a}\left(\frac{\dot{a}^{2}+k}{a^{2}}\right)=-p^{(h)} \\
& \left(\frac{\dot{a}^{2}+k}{a^{2}}\right)\left[(h-h(0)) \frac{\dot{a}}{a}+\dot{h}\right]=0
\end{aligned}
$$

and the conservation (34) (divergence-free energy-momentum tensor) for each of the fluids are given by

$\dot{\rho}+4 \frac{\dot{a}}{a} \rho=0$

and

$\dot{\rho}^{(h)}+4 \frac{\dot{a}}{a}\left(\rho^{(h)}+p^{(h)}\right)=0$.

Equation (96) has as solution

$\rho(t)=\left(\frac{a_{0}}{a(t)}\right)^{4} \rho_{0}$

where the initial conditions $a_{0}=a\left(t_{0}\right)$ and $\rho_{0}=\rho\left(t_{0}\right)$ have been set.

Substituting (98) and (93) into (91) we have

$$
\left(\frac{\dot{a}^{2}+k}{a^{2}}\right)^{2}-2 A\left(\frac{\dot{a}^{2}+k}{a^{2}}\right)+A B \frac{a_{0}^{4}}{a^{4}}=0
$$

where we defined

$$
A:=\frac{1}{\alpha l^{2}}, \quad B:=\frac{\kappa_{5} \rho_{0}}{3} \text {. }
$$

\subsection{Case $k=-1$}

In this case, (99) can be rewritten

$$
\left(\frac{\dot{a}^{2}-1}{a^{2}}\right)^{2}-2 A\left(\frac{\dot{a}^{2}-1}{a^{2}}\right)+A B \frac{a_{0}^{4}}{a^{4}}=0
$$

where we find

$\dot{a}= \pm \sqrt{A a^{2}\left(1 \pm \operatorname{sgn}(A) \sqrt{1-\frac{B}{A} \frac{a_{0}^{4}}{a^{4}}}\right)+1}$.

\subsubsection{Case $\alpha>0$}

In this case

$A=\frac{1}{\alpha l^{2}}>0$.

From (102) we can see that $\dot{a}$ is well defined if

$a \geq a_{\min }=\sqrt[4]{\frac{B}{A}} a_{0}$

where

$a_{\min }=\sqrt[4]{\frac{\kappa_{5} \alpha l^{2} \rho_{0}}{3}} a_{0}$.

On the other hand $a_{0}$ must satisfy

$a_{0} \geq a_{\min }$

so that

$\frac{B}{A} \leq 1$ i.e., $B \leq A$

and therefore

$\rho_{0} \leq \rho_{\max }=\frac{3}{\kappa_{5} \alpha l^{2}}$.

These results allow us to analyze the radicand in (102)

$A a^{2}\left(1 \pm \sqrt{1-\frac{a_{\mathrm{min}}^{4}}{a^{4}}}\right)+1 \geq 0$,

i.e.,

$-A^{2} a_{\min }^{4} \leq 1+2 A a^{2}$,

which is satisfied for all $a$.

a. Plus or minus sign?

The choice of the sign in the radicand has information as regards the allowed values of $\dot{a}$. Let us consider $\dot{a}>0$ (the analysis of the case $\dot{a}<0$ is very similar)

$\dot{a}=\sqrt{A a^{2}\left(1 \pm \sqrt{1-\frac{a_{\mathrm{min}}^{4}}{a^{4}}}\right)-k}$.

The function $\dot{a}(a)$ is monotonically increasing (decreasing) if we consider the plus (minus) sign in front of the square root. 
From (110) we can see that there exists $\dot{a}_{\text {cri }}$

$\dot{a}_{\mathrm{cri}}:=\dot{a}\left(a_{\min }^{4}\right)=\sqrt{\sqrt{\frac{\kappa_{5} \rho_{0}}{3 \alpha l^{2}}} a_{0}^{2}-k}$.

If we consider the plus (minus) sign in front of the square root, $\dot{a}_{\text {cri }}$ is the minimum (maximum) value of $\dot{a}$.

If there is a limit to $a \gg a_{\min }$, then

$$
\begin{aligned}
\dot{a} & = \pm \sqrt{A a^{2}\left(1 \pm \sqrt{\left.1-\frac{a_{\mathrm{min}}^{4}}{a^{4}}\right)-k}\right.} \\
& \approx \pm \sqrt{A a^{2}\left\{1 \pm\left(1-\frac{a_{\mathrm{min}}^{4}}{2 a^{4}}\right)\right\}-k}
\end{aligned}
$$

where $k=-1$.

b. Case where the sign is "+".

In this case

$\dot{a}= \pm \sqrt{A a^{2}\left(2-\frac{a_{\text {min }}^{4}}{2 a^{4}}\right)-k} \approx \pm \sqrt{2 A a^{2}-k}$,

whose approximate solution is

$$
\begin{aligned}
a(t)= & \pm \sqrt{-\frac{\alpha l^{2} k}{2}} \\
& \times \sin h\left[\sqrt{\frac{2}{\alpha l^{2}}}\left(t-t_{0}\right)+\operatorname{arsinh}\left(\sqrt{-\frac{2}{\alpha l^{2} k}} a_{0}\right)\right]
\end{aligned}
$$

where we use $A=\frac{1}{\alpha l^{2}}$ and $k=-1$.

b. Case where the sign is "-".

In this case

$\dot{a}= \pm \sqrt{A \frac{a_{\mathrm{min}}^{4}}{2 a^{2}}-k} \approx \pm \sqrt{-k}$

whose approximate solution is

$a(t)= \pm \sqrt{-k}\left(t-t_{0}\right)+a_{0}$

where we use $k=-1$.

\subsubsection{Case $\alpha<0$}

In this case

$A=\frac{1}{\alpha l^{2}}<0$.
From (102) we can see that $\dot{a}$ is well defined if

$1-\frac{B}{A} \frac{a_{0}^{4}}{a^{4}} \geq 0$

but this condition is satisfied for all $a$.

a. Case where the sign is "+".

In this case

$A a^{2}\left(1+\sqrt{1-\frac{B a_{0}^{4}}{A a^{4}}}\right)-k \geq 0$,

so that

$\frac{k-A a^{2}}{A a^{2}} \geq \sqrt{1-\frac{B a_{0}^{4}}{A a^{4}}}$

The left side of the last equation must be positive, i.e.,

$k-A a^{2} \leq 0$ or $a \leq \sqrt{\frac{k}{A}}$.

From (119) we obtain

$k^{2}-2 A k a^{2} \geq-A B a_{0}^{4}$

and again, the left side of the last equation must be positive, i.e.,

$k^{2}-2 A k a^{2} \geq 0 \Longleftrightarrow a \leq \sqrt{\frac{k}{2 A}}$

and from (121) we find

$a \leq \sqrt{\frac{k^{2}+A B a_{0}^{4}}{2 A k}}$.

Since

$\sqrt{\frac{k}{A}}>\sqrt{\frac{k}{2 A}}>\sqrt{\frac{k^{2}+A B a_{0}^{4}}{2 A k}}=a_{\max } \geq a$,

we have found a maximum value for $a$

$a_{\max }=\sqrt{\frac{3 \alpha l^{2} k^{2}+\kappa_{5} \rho_{0} a_{0}^{4}}{6 k}}$

and therefore

$\dot{a}\left(a=a_{\text {max }}\right)=0$

i.e., $a_{\max }$ is a local maximum. It is straightforward to prove that $\dot{a} \neq 0$ for $a \neq a_{\max }$. If $a$ has a maximum value $a_{\max }$ then (see (98))

$\rho(t)=\left(\frac{a_{0}}{a(t)}\right)^{4} \rho_{0} \geq\left(\frac{a_{0}}{a_{\max }}\right)^{4} \rho_{0}=\rho_{\min }$. 
This means that $\rho$ has a minimum value $\rho_{\text {min }}$ given by

$\rho_{\min }=\left(\frac{6 k a_{0}^{2}}{3 \alpha l^{2} k^{2}+\kappa_{5} \rho_{0} a_{0}^{4}}\right)^{2} \rho_{0}$

where $k=-1$.

Consider the case where $\dot{a}>0$. We just consider $\dot{a}>0$ because the analysis of the case $\dot{a}<0$ looks very similar. In this case

$\dot{a}=\sqrt{A a^{2}\left(1+\sqrt{1-\frac{B a_{0}^{4}}{A a^{4}}}\right)-k}$

is a decreasing function. We can see that the minimum value of $\dot{a}$ is given by

$\dot{a}_{\min }=\dot{a}\left(a_{\max }\right)=0$

and the maximum value of $\dot{a}$ is given by

$\dot{a}_{\max }=\dot{a}(a=0)=\sqrt{-\sqrt{-\frac{\kappa_{5} \rho_{0}}{3 \alpha l^{2}}} a_{0}^{2}-k}$.

b. Case where the sign is "-".

In this case we obtain the following condition:

$A a^{2}\left(1-\sqrt{1-\frac{B a_{0}^{4}}{A a^{4}}}\right)-k \geq 0$

where $A=-\frac{1}{\alpha l^{2}}$ and $k=-1$. This condition is trivially satisfied for all $a$.

This result implies that $\dot{a} \neq 0$. This means that $a$ has no local maxima/minima, so $a$ is monotonically increasing or monotonically decreasing.

If there is a limit to $a \gg \sqrt[4]{-\frac{B}{A}} a_{0}$, then

$$
\begin{aligned}
\dot{a} & = \pm \sqrt{A a^{2}\left(1-\sqrt{1-\frac{B a_{0}^{4}}{A a^{4}}}\right)-k} \\
& \approx \pm \sqrt{A a^{2}\left(1-\left(1-\frac{B a_{0}^{4}}{2 A a^{4}}\right)\right)-k}
\end{aligned}
$$

and

$\dot{a}= \pm \sqrt{\frac{B a_{0}^{4}}{2 a^{2}}-k} \approx \pm \sqrt{-k}$,

whose approximate solution is

$a(t)= \pm \sqrt{-k}\left(t-t_{0}\right)+a_{0}$

where we use $k=-1$.
In this case

$\dot{a}=\sqrt{A a^{2}\left(1-\sqrt{1-\frac{B a_{0}^{4}}{A a^{4}}}\right)-k}$

is a decreasing function. The maximum value of $\dot{a}$ is given by

$\dot{a}_{\max }=\dot{a}(a=0)=\sqrt{\sqrt{-\frac{\kappa_{5} \rho_{0}}{3 \alpha l^{2}}} a_{0}^{2}-k}$

and we can see that $\dot{a}$ tends to a minimum value given by

$\dot{a}_{\min }=\dot{a}(a \rightarrow \infty)=\sqrt{-k}=1$.

6.2 Case $k=0$

In this case, (99) takes the form

$\left(\frac{\dot{a}}{a}\right)^{4}-2 A\left(\frac{\dot{a}}{a}\right)^{2}+A B \frac{a_{0}^{4}}{a^{4}}=0$

from which

$\dot{a}= \pm \sqrt{A a^{2}\left(1 \pm \operatorname{sgn}(A) \sqrt{1-\frac{B}{A} \frac{a_{0}^{4}}{a^{4}}}\right)}$.

\subsubsection{Case $\alpha>0$}

In this case

$A=\frac{1}{\alpha l^{2}}>0$.

From (139) we can see that $\dot{a}$ is well defined if

$a \geq \sqrt[4]{\frac{B}{A}} a_{0}$

and therefore a minimum value for $a$ is given by

$a_{\min }=\sqrt[4]{\frac{\kappa_{5} \alpha l^{2} \rho_{0}}{3}} a_{0}$.

On the other hand $a_{0} \geq a_{\min }$, so that

$B \leq A$ i.e., $\rho_{0} \leq \rho_{\max }=\frac{3}{\kappa_{5} \alpha l^{2}}$.

These results lead to

$A a^{2}\left(1 \pm \operatorname{sgn}(A) \sqrt{1-\frac{B}{A} \frac{a_{0}^{4}}{a^{4}}}\right) \geq 0$,

i.e., $a$ has no local maxima/minima, ${ }^{2}$ so that $a$ is monotonically increasing or monotonically decreasing.

2 Only it has a local maximum/minimum if we consider the minus sign in the radicand. In that case the local minimum is $a_{\min }$. We can prove that there is no local maximum. 
Plus or minus sign?

The choice of the sign into the radicand has information as regards the allowed values of $\dot{a}$. Let us consider $\dot{a}>0$ (the analysis of the case $\dot{a}<0$ is very similar):

$\dot{a}=\sqrt{A a^{2}\left(1 \pm \sqrt{1-\frac{a_{\mathrm{min}}^{4}}{a^{4}}}\right)}$.

The function $\dot{a}(a)$ is monotonically increasing (decreasing) if we consider the plus (minus) sign in front of the square root.

From (145) we can see that there exists $\dot{a}_{\text {cri }}$

$\dot{a}_{\mathrm{cri}}:=\dot{a}_{\mathrm{min}}=\sqrt{A} a_{\mathrm{min}}=\sqrt[4]{\frac{\kappa 5 \rho_{0}}{3 \alpha l^{2}}} a_{0}$.

If we consider the plus (minus) sign in front of the square root, $\dot{a}_{\text {cri }}$ is the minimum (maximum) value of $\dot{a}$.

If there is a limit to $a \gg a_{\min }$ then

$$
\begin{aligned}
\dot{a} & = \pm \sqrt{A a^{2}\left(1 \pm \sqrt{1-\frac{a_{\mathrm{min}}^{4}}{a^{4}}}\right)} \\
& \approx \pm a \sqrt{A\left(1 \pm\left(1-\frac{a_{\mathrm{min}}^{4}}{2 a^{4}}\right)\right)} .
\end{aligned}
$$

a. Case where the sign is "+".

In this case

$\dot{a}= \pm a \sqrt{A\left(2-\frac{a_{\mathrm{min}}^{4}}{2 a^{4}}\right)} \approx \pm a \sqrt{2 A}$,

whose approximate solution is

$a(t)=a_{0} \exp \left( \pm \sqrt{\frac{2}{\alpha l^{2}}}\left(t-t_{0}\right)\right)$

where $A=\frac{1}{\alpha l^{2}}>0$.

b. Case where the sign is "-".

In this case

$\dot{a} \approx \pm \sqrt{\frac{A}{2}} \frac{a_{\mathrm{min}}^{2}}{a}$,

whose approximate solution is

$$
\begin{aligned}
a(t) & = \pm \sqrt{a_{0}^{2} \pm \sqrt{\frac{2}{\alpha l^{2}} a_{\min }^{2}\left(t-t_{0}\right)}} \\
& = \pm a_{0} \sqrt{1 \pm \sqrt{\frac{2 \kappa_{5} \rho_{0}}{3 a_{0}^{4}}}\left(t-t_{0}\right)}
\end{aligned}
$$

where we use $A=\frac{1}{\alpha l^{2}}>0$ and $a_{\min }=\sqrt[4]{\frac{\kappa_{5} \alpha l^{2} \rho_{0}}{3}} a_{0}$.
6.2.2 Case $\alpha<0$

In this case

$A=\frac{1}{\alpha l^{2}}<0$.

From (139) we can see that $\dot{a}$ is well defined if

$1 \mp \sqrt{1-\frac{B}{A} \frac{a_{0}^{4}}{a^{4}}} \leq 0$.

This condition is only satisfied if we use the minus sign "-_" for all $a$, i.e.,

$1-\sqrt{1-\frac{B}{A} \frac{a_{0}^{4}}{a^{4}}}<0$,

and therefore $a$ has no local maxima/minima, so $a$ is monotonically increasing or monotonically decreasing. Thus, $\dot{a}$ has a maximum value in $a=0$, i.e.,

$\dot{a}_{\max }=\dot{a}(a=0)=\sqrt[4]{-\frac{\kappa_{5} \rho_{0}}{3 \alpha l^{2}}} a_{0}$,

and $\dot{a}$ tends to a minimum value given by

$\dot{a}_{\min }=\dot{a}(a \rightarrow \infty)=0$.

If there exists a limit for $a \gg \sqrt[4]{-\frac{B}{A}} a_{0}$ then

$\dot{a}= \pm \sqrt{A a^{2}\left(1-\sqrt{\left.1-\frac{B}{A} \frac{a_{0}^{4}}{a^{4}}\right)}\right.} \approx \pm \sqrt{\frac{B}{2}} \frac{a_{0}^{2}}{a}$,

whose approximate solution is

$a(t)=a_{0} \sqrt{1 \pm \sqrt{\frac{2 \kappa_{5} \rho_{0}}{3 a_{0}^{4}}}\left(t-t_{0}\right)}$

where we use $B=\frac{\kappa_{5} \rho_{0}}{3}$.

6.3 Case $k=1$

In this case, (99) can be rewritten as

$\left(\frac{\dot{a}^{2}+1}{a^{2}}\right)^{2}-2 A\left(\frac{\dot{a}^{2}+1}{a^{2}}\right)+A B \frac{a_{0}^{4}}{a^{4}}=0$,

from which

$\dot{a}= \pm \sqrt{A a^{2}\left(1 \pm \operatorname{sgn}(A) \sqrt{1-\frac{B}{A} \frac{a_{0}^{4}}{a^{4}}}\right)-k}$

with $k=1$. 


\subsubsection{Case $\alpha>0$}

In this case

$A=\frac{1}{\alpha l^{2}}>0$.

From (159) we can see that $\dot{a}$ is well defined if

$a_{\min }=\sqrt[4]{\frac{\kappa_{5} \alpha l^{2} \rho_{0}}{3}} a_{0}$,

so that

$B \leq A \quad$ i.e., $\quad \rho_{0} \leq \rho_{\max }=\frac{3}{\kappa_{5} \alpha l^{2}}$.

With these considerations we can analyze if the radicand is positive in (159):

$A a^{2}\left(1 \pm \sqrt{1-\frac{a_{\min }^{4}}{a^{4}}}\right)-k$.

\section{a. Plus or minus sign?}

Let us consider $\dot{a}>0$ (the analysis of the case $\dot{a}<0$ is very similar):

$\dot{a}=\sqrt{A a^{2}\left(1 \pm \sqrt{1-\frac{a_{\mathrm{min}}^{4}}{a^{4}}}\right)-k}$.

The function $\dot{a}(a)$ is monotonically increasing (decreasing) if we consider the plus (minus) sign in front of the square root.

From (164) we can see that there exists $\dot{a}_{\text {cri }}$

$\dot{a}_{\text {cri }}:=\dot{a}\left(a_{\min }\right)=\sqrt{\sqrt{\frac{\kappa_{5} \rho_{0}}{3 \alpha l^{2}}} a_{0}^{2}-k}$.

If we consider the plus (minus) sign in front of the square root, $\dot{a}_{\text {cri }}$ is the minimum (maximum) value of $\dot{a}$.

b. Case where the sign is "+".

In this case

$A a^{2}\left(1+\sqrt{1-\frac{a_{\text {min }}^{4}}{a^{4}}}\right)-k \geq A a_{\min }^{2}-k \geq 0$,

so that

$a_{\min } \geq \sqrt{\frac{k}{A}} \Longleftrightarrow \rho_{0} a_{0}^{4} \geq 3 \frac{\alpha l^{2} k^{2}}{\kappa_{5}}$,

but (see (98))

$\rho(t)=\left(\frac{a_{0}}{a(t)}\right)^{4} \rho_{0} \Longrightarrow \rho a^{4}=\rho_{0} a_{0}^{4}$, then

$\rho a^{4} \geq 3 \frac{\alpha l^{2} k^{2}}{\kappa_{5}}$.

It is straightforward to prove that $\dot{a} \neq 0$ for $a>a_{\min }$, then $a$ has no local maxima/minima, and therefore $a$ is monotonically increasing or monotonically decreasing.

If there is a limit to $a \gg a_{\min }$, then

$\dot{a}= \pm \sqrt{A a^{2}\left(1+\sqrt{1-\frac{a_{\min }^{4}}{a^{4}}}\right)-k} \approx \pm \sqrt{2 A a^{2}-k}$,

whose approximate solution is

$$
\begin{aligned}
a(t)= & \pm \sqrt{\frac{\alpha l^{2} k}{2}} \\
& \times \cos h\left[\sqrt{\frac{2}{\alpha l^{2}}}\left(t-t_{0}\right)+\operatorname{arcos} h\left(\sqrt{\frac{2}{\alpha l^{2} k}} a_{0}\right)\right]
\end{aligned}
$$

where we use $A=\frac{1}{\alpha l^{2}}$ and $k=1$.

c. Case where the sign is "-".

In this case

$A a^{2}\left(1-\sqrt{1-\frac{a_{\min }^{4}}{a^{4}}}\right)-k \geq 0$,

therefore

$\frac{A a^{2}-k}{A a^{2}} \geq \sqrt{1-\frac{a_{\mathrm{min}}^{4}}{a^{4}}}$.

This condition must also be satisfied by $a_{\min }$

$A a_{\min }^{2}-k \geq 0 \Longleftrightarrow a_{\min } \geq \sqrt{\frac{k}{A}}$,

so that

$\rho_{0} a_{0}^{4} \geq 3 \frac{\alpha l^{2} k^{2}}{\kappa_{5}}$,

but (see (98))

$\rho a^{4}=\rho_{0} a_{0}^{4}$

and therefore

$\rho a^{4} \geq 3 \frac{\alpha l^{2} k^{2}}{\kappa_{5}}$.

From (171) we obtain

$a \leq a_{\max }=\sqrt{\frac{k^{2}+A^{2} a_{\min }^{4}}{2 A k},}$ 
i.e.,

$a_{\max }=\sqrt{\frac{3 \alpha l^{2} k^{2}+\kappa_{5} \rho_{0} a_{0}^{4}}{6 k}}$.

From (176) we have

$\rho=\frac{a_{0}^{4}}{a^{4}} \rho_{0}$,

from which

$\rho_{\min }=\frac{a_{0}^{4}}{a_{\max }^{4}} \rho_{0}$

and therefore

$\rho_{\min }=\left(\frac{6 k a_{0}^{2}}{3 \alpha l^{2} k^{2}+\kappa_{5} \rho_{0} a_{0}^{4}}\right)^{2} \rho_{0}$.

\subsubsection{Case $\alpha<0$}

In this case

$A=\frac{1}{\alpha l^{2}}<0$.

From (159) we can see that $\dot{a}$ is well defined if

$A a^{2}\left(1 \pm \operatorname{sgn}(A) \sqrt{1-\frac{B}{A} \frac{a_{0}^{4}}{a^{4}}}\right)-k \geq 0$.

This constraint excludes the case with the plus sign "+" in front of the square root. This condition leads to

$a \leq a_{\max }=\sqrt{\frac{-A B a_{0}^{4}-k^{2}}{-2 A k}}$

where $k=1$ and $A=\frac{1}{\alpha l^{2}}<0$. There is a maximum value for $a$ :

$a_{\max }=\sqrt{\frac{3 \alpha l^{2} k^{2}+\kappa_{5} \rho_{0} a_{0}^{4}}{6 k}}$,

this maximum leads to

$\rho_{0} a_{0}^{4} \geq-3 \frac{\alpha l^{2} k^{2}}{\kappa_{5}}$,

but (see (98))

$\rho a^{4}=\rho_{0} a_{0}^{4}$,

so that

$\rho a^{4} \geq-3 \frac{\alpha l^{2} k^{2}}{\kappa_{5}}$.

If there is a maximum $a_{\max }$ then there must exist a minimum for $\rho$

$\rho_{\min }=\left(\frac{6 k a_{0}^{2}}{3 \alpha l^{2} k^{2}+\kappa_{5} \rho_{0} a_{0}^{4}}\right)^{2} \rho_{0}$.

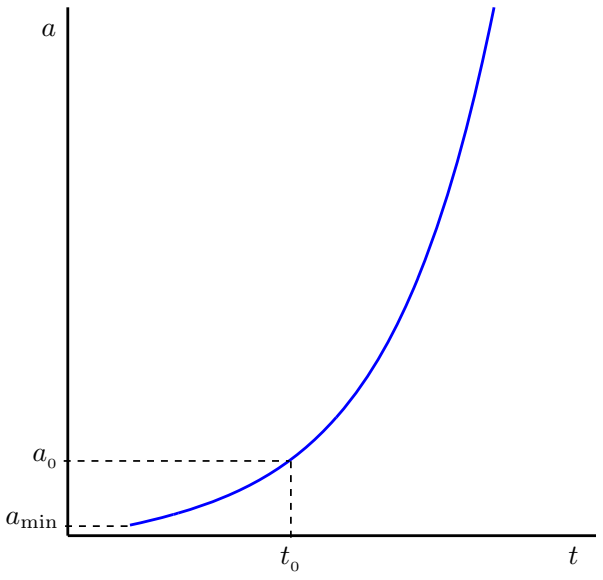

Fig. 4 Numerical solution with $A>0, k=-1$, and $\dot{a}_{0}>\dot{a}_{\text {cri }}$ of $\dot{a}=\sqrt{A a^{2}\left(1+\sqrt{1-\frac{a_{\min }^{4}}{a^{4}}}\right)-k}$

There is no a limit to $a \longrightarrow \infty$ and therefore it is impossible to find an approximate solution for

$\dot{a}= \pm \sqrt{A a^{2}\left(1-\sqrt{1-\frac{B}{A} \frac{a_{0}^{4}}{a^{4}}}\right)-k}$.

\subsection{Solutions for era of matter}

We have found a family of solutions for the era of matter.

If we consider an open space $(k=-1)$, the solutions found include (i) an accelerated expansion $(\alpha>0)$ with a minimum scale factor at initial time such that, when time goes to infinity, the scale factor behaves as a hyperbolic sine function (Fig. 4), (ii) a decelerated expansion $(\alpha<0)$, with a Big Crunch in a finite time $t_{\max }$ (Fig. 5), and (iii) a couple of solutions without accelerated expansion, whose scale factor tends to a constant value: $\alpha>0$ (Fig. 6) and $\alpha<0$ (Fig. 7). See Tables 4 and 5 .

From models found in Sect. 6.1 we can see that there are solutions with $\alpha>0$ for an accelerated contracting universe and no accelerated contracting universe (see Fig. 8, $\dot{a}<0$ ). These solutions were not studied.

Solutions found for a flat universe $(k=0)$ in expansion are (i) an accelerated expansion whose scale factor behaves as an exponential function when time grows and starts from a minimum value (Fig. 9), and (ii) a couple of solutions with decelerated expansion whose scale factor tends to a square root function: $\alpha>0$ (Fig. 10) and $\alpha<0$ (Fig. 11). See Tables 6 and 7.

In this case there are also solutions of a contraction universe $(\dot{a}<0)$ : (i) one ends with a minimum value $a_{\min }$ when $\alpha$ is positive (Fig. 12), and (ii) the other ends with a Big Crunch when $\alpha$ is negative (Fig. 13). 


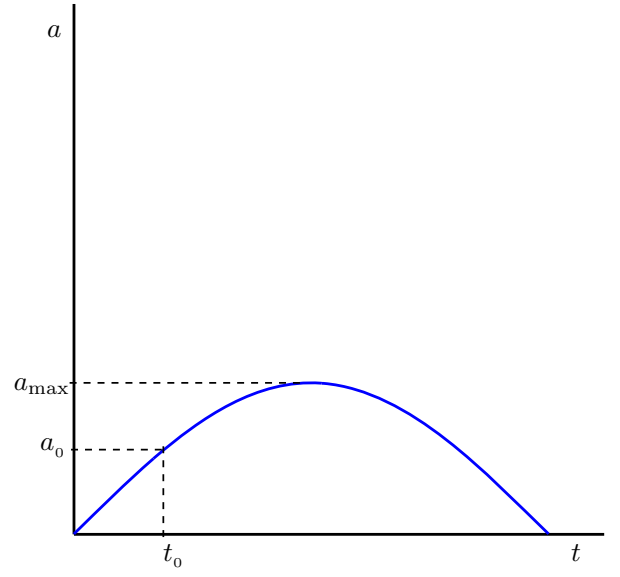

Fig. 5 Solution of $\dot{a}=\sqrt{A a^{2}\left(1-\sqrt{1-\frac{B a_{0}^{4}}{A a^{4}}}\right)-k}$ with $A<0$, $k=-1$, and $\left|\dot{a}_{0}\right|<\dot{a}_{\max }$

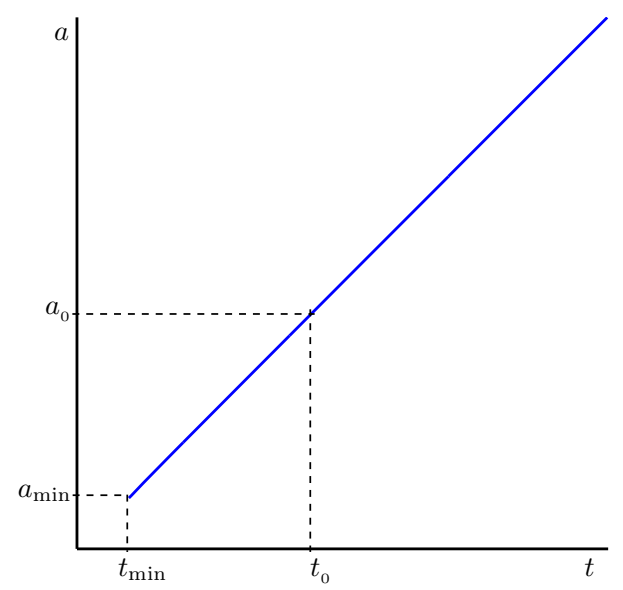

Fig. 6 Numerical solution with $A>0, k=-1$, and $\dot{a}_{0}<\dot{a}_{\text {cri }}$ of $\dot{a}=\sqrt{A a^{2}\left(1+\sqrt{1-\frac{a_{\min }^{4}}{a^{4}}}\right)-k}$

Finally, we only found one solution for a closed universe $(k=1)$ in expansion. This solution is found when $\alpha$ is greater than zero. It behaves as a hyperbolic cosine function when time grows and starts from a minimum value (Fig. 14). See Table 8.

Furthermore, there are two contracting universe solutions, both ends in a finite time: (i) one ends with a minimum value $a_{\text {min }}$, when $\alpha$ is positive (Fig. 15), and (ii) the other ends with a Big Crunch, when $\alpha$ is negative (see Table 9; Fig. 16).

\section{Summary}

We have considered a five-dimensional Einstein-ChernSimons action $S=S_{g}+S_{M}$, which is composed of a gravitational sector and a sector of matter, where the gravitational sector is given by a Chern-Simons gravity action instead of

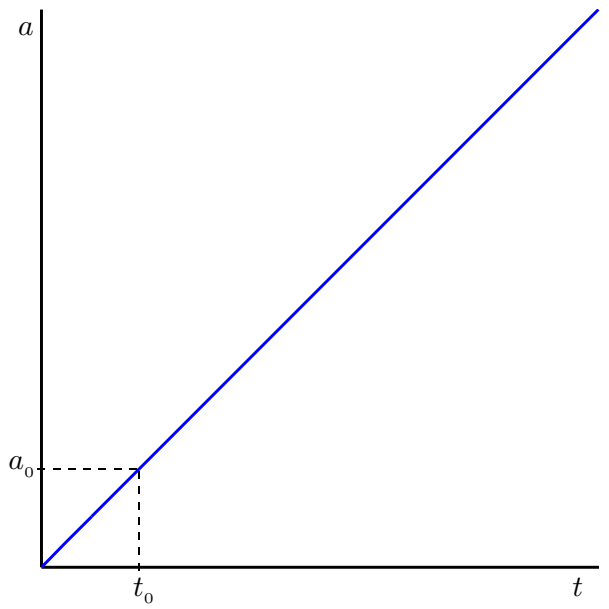

Fig. 7 Solution of $\dot{a}=\sqrt{A a^{2}\left(1-\sqrt{1-\frac{B a_{0}^{4}}{A a^{4}}}\right)-k}$ with $A<0$, $k=-1$, and $1<\dot{a}_{0}<\dot{a}_{\max }$

Table 4 Expanding universe solutions for scale factor of an open space $k=-1$ (hyperbolic) with $\alpha>0$, where $a_{\min }=\sqrt[4]{\frac{\kappa_{5} \alpha l^{2} \rho_{0}}{3}} a_{0}, \omega=$ $\sqrt{\frac{2}{\alpha l^{2}}}, \phi=\operatorname{arsinh}\left(\frac{\omega}{\sqrt{-k}} a_{0}\right), \rho_{\max }=\frac{3}{\kappa_{5} \alpha l^{2}}$, and $\dot{a}_{\mathrm{cri}}=\sqrt{\sqrt{\frac{\kappa_{5} \rho_{0}}{3 \alpha l^{2}}} a_{0}^{2}-k}$

\begin{tabular}{lll}
\hline & Accelerated & No accelerated \\
\hline$a$ & $a_{\min } \leq a$ & $a_{\min } \leq a$ \\
$a(t \rightarrow \infty)$ & $\sim \sin h\left(\omega\left(t-t_{0}\right)+\phi\right)$ & $\sim\left(t-t_{0}\right)$ \\
$\rho \sim \frac{1}{a^{4}}$ & $0<\rho \leq \rho_{\max }$ & $0<\rho \leq \rho_{\max }$ \\
$\dot{a}$ & $\dot{a}_{\mathrm{cri}}<\dot{a}$ & $\sqrt{-k}<\dot{a}<\dot{a}_{\mathrm{cri}}$ \\
$\dot{a}(t \rightarrow \infty)$ & $\sim \cosh \left(\omega\left(t-t_{0}\right)+\phi\right)$ & $\sim \sqrt{-k}$ \\
\hline
\end{tabular}

Table 5 Expanding universe solutions for the scale factor of an open space $k=-1$ (hyperbolic) with $\alpha<0$, where $a_{\max }=\sqrt{\frac{3 \alpha l^{2} k^{2}+\kappa_{5} \rho_{0} a_{0}^{4}}{6 k}}$, $\rho_{\min }=\left(\frac{6 k a_{0}^{2}}{3 \alpha l^{2} k^{2}+\kappa_{5} \rho_{0} a_{0}^{4}}\right)^{2} \rho_{0}$, and $\dot{a}_{\max \pm}=\sqrt{ \pm \sqrt{-\frac{\kappa_{5} \rho_{0}}{3 \alpha l^{2}}} a_{0}^{2}-k}$. A decelerated solution describes a Big Crunch in a finite time $t_{\max }$

\begin{tabular}{lll}
\hline & Decelerated & No accelerated \\
\hline$a$ & $0 \leq a \leq a_{\max }$ & $0 \leq a$ \\
$a(t \rightarrow \infty)$ & - & $\sim\left(t-t_{0}\right)$ \\
$\rho \sim \frac{1}{a^{4}}$ & $\rho_{\min } \leq \rho$ & $0<\rho$ \\
$\dot{a}$ & $-\dot{a}_{\max -} \leq \dot{a} \leq \dot{a}_{\max -}$ & $\sqrt{-k}<\dot{a} \leq \dot{a}_{\max }+$ \\
$\dot{a}(t \rightarrow \infty)$ & - & $\sim \sqrt{-k}$ \\
\hline
\end{tabular}

the Einstein-Hilbert action and where the matter sector is given by a so-called perfect fluid. We have shown that:

i The Einstein-Chern-Simons field equations (9)-(12) subject to the conditions $T^{a}=0, k^{a b}=0$, and $\frac{\delta L_{M}}{\delta \omega^{a b}}=0$ are rewritten in a way similar to the Einstein-Maxwell field equations (20)-(22). In the case where (20)-(22) 




Fig. 8 For every $a_{0}$ allowed there are two different values for $\dot{a}>$ 0 : evolution with $\dot{a}$ approximate constant and evolution accelerated (decelerated)

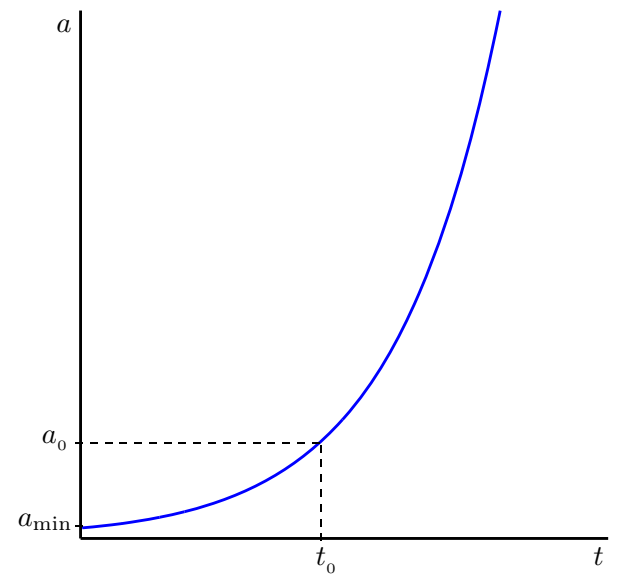

Fig. 9 Solution of $\dot{a}=\sqrt{A a^{2}\left(1+\sqrt{1-\frac{a_{\min }^{4}}{a^{4}}}\right)}$ with $A>0$ and $\dot{a}_{0}>\dot{a}_{\text {cri }}$

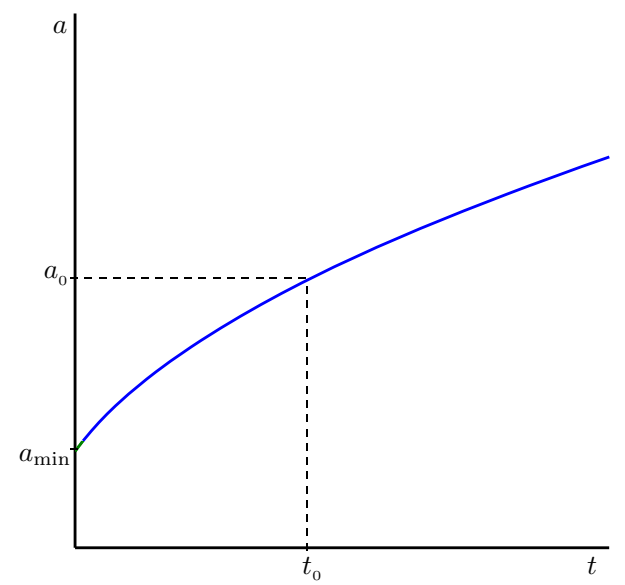

Fig. 10 Solution of $\dot{a}=\sqrt{A a^{2}\left(1-\sqrt{1-\frac{a_{\min }^{4}}{a^{4}}}\right)}$ with $A>0$ and $\dot{a}_{0}<\dot{a}_{\text {cri }}$

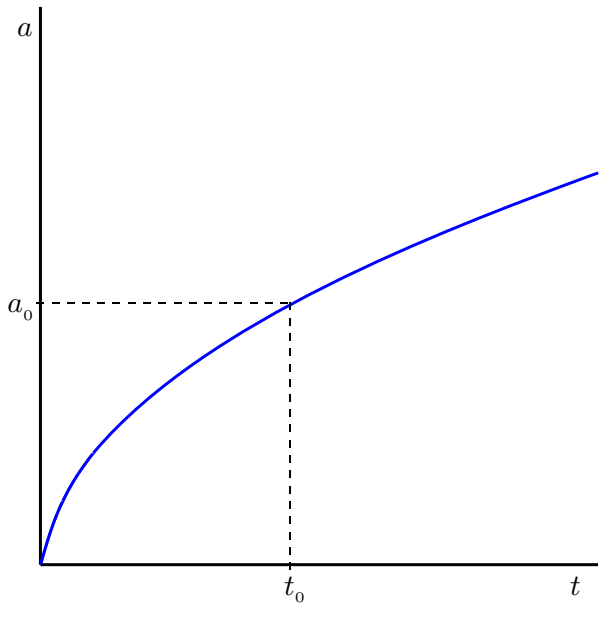

Fig. 11 Solution of $\dot{a}=\sqrt{A a^{2}\left(1-\sqrt{1-\frac{B a_{0}^{4}}{A a^{4}}}\right)}$ with $A<0, k=0$, and $\dot{a}_{0}<\dot{a}_{\max }$

Table 6 Expanding universe solutions for scale factor of a flat space $k=0$ with $\alpha>0$, where $a_{\min }=\sqrt[4]{\frac{\kappa_{5} \alpha l^{2} \rho_{0}}{3}} a_{0}, \omega=\sqrt{\frac{2}{\alpha l^{2}}}, \rho_{\max }=$ $\frac{3}{\kappa_{5} \alpha l^{2}}$, and $\dot{a}_{\mathrm{cri}}=\sqrt[4]{\frac{\kappa_{5} \rho_{0}}{3 \alpha l^{2}}} a_{0}$

\begin{tabular}{lll}
\hline & Accelerated & Decelerated \\
\hline$a$ & $a_{\min } \leq a$ & $a_{\min } \leq a$ \\
$a(t \rightarrow \infty)$ & $\sim \exp \left(\omega\left(t-t_{0}\right)\right)$ & $\sim \sqrt{1+\omega\left(a_{\min } / a_{0}\right)^{2}\left(t-t_{0}\right)}$ \\
$\rho \sim \frac{1}{a^{4}}$ & $0<\rho \leq \rho_{\max }$ & $0<\rho \leq \rho_{\max }$ \\
$\dot{a}$ & $\dot{a}_{\text {cri }}<\dot{a}$ & $0<\dot{a}<\dot{a}_{\text {cri }}$ \\
$\dot{a}(t \rightarrow \infty)$ & $\sim \exp \left(\omega\left(t-t_{0}\right)\right)$ & $\sim \frac{1}{\sqrt{1+\omega\left(a_{\min } / a_{0}\right)^{2}\left(t-t_{0}\right)}}$ \\
\hline
\end{tabular}

Table 7 Expanding universe solutions for scale factor of a flat space $k=0$ with $\alpha<0$, where $a_{\text {ref }}=\sqrt[4]{-\frac{\kappa_{5} \alpha l^{2} \rho_{0}}{3}} a_{0}, \omega=\sqrt{-\frac{2}{\alpha l^{2}}}$, and $\dot{a}_{\max }=\sqrt[4]{-\frac{\kappa 5 \rho_{0}}{3 \alpha l^{2}}} a_{0}$

\begin{tabular}{ll}
\hline & Decelerate \\
\hline$a$ & $0 \leq a$ \\
$a(t \rightarrow \infty)$ & $\sim \sqrt{1+\omega\left(a_{\text {ref }} / a_{0}\right)^{2}\left(t-t_{0}\right)}$ \\
$\rho \sim \frac{1}{a^{4}}$ & $0 \leq \rho$ \\
$\dot{a}$ & $0<\dot{a} \leq \dot{a}_{\max }$ \\
$\dot{a}(t \rightarrow \infty)$ & $\sim \frac{1}{\sqrt{1+\omega\left(a_{\mathrm{ref}} / a_{0}\right)^{2}\left(t-t_{0}\right)}}$ \\
\hline
\end{tabular}

satisfy the cosmological principle and the ordinary matter is negligible compared to the dark energy, we find that (20)-(22) take the form (41)-(45). When ordinary matter is modeled as dust (era of matter), we find that (20)-(22) take the form (91)-(95).

ii The field equations (41)-(45) were completely resolved for the age of dark energy (Sect. 5, accelerated expansion). We find that the field $h^{a}$ has a similar behavior to that of a cosmological constant. 


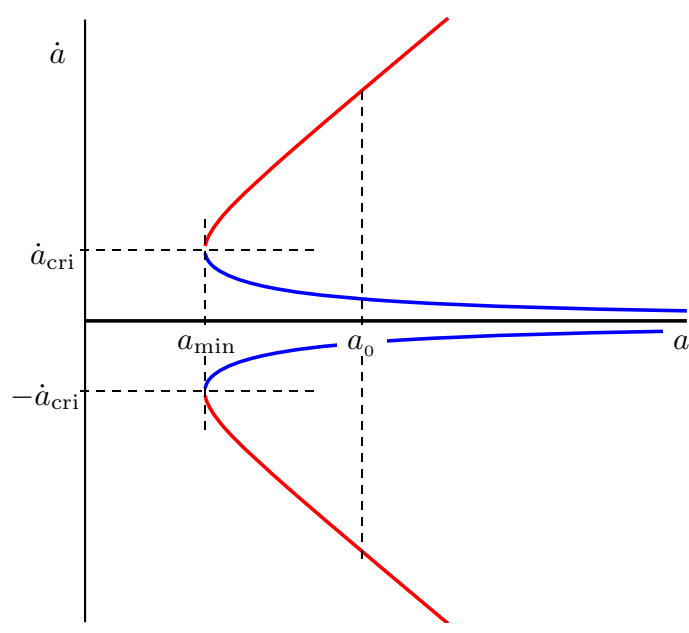

Fig. 12 For every $a_{0}$ there are two different values for $\dot{a}$ : evolution with $\dot{a}<\dot{a}_{\text {cri }}$ and expansion accelerated (decelerated) with $|\dot{a}|>\left|\dot{a}_{\text {cri }}\right|$

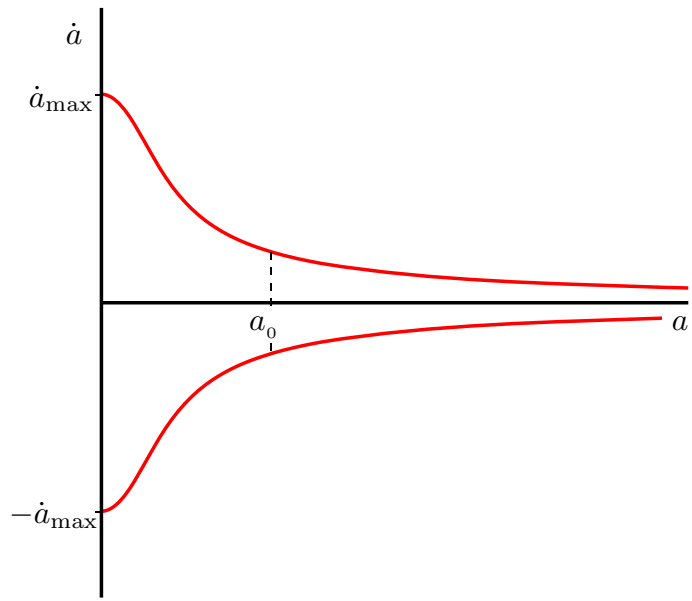

Fig. 13 Phase space for $A<0$ and $k=0$ with “-” sign

iii The field equations (91)-(95) were solved for the era of matter (Sect. 6). We find several models that are consistent with standard cosmology. The dynamics of the field $h^{a}$ (95) was not analyzed because the focus was placed on the dynamics of the scale factor $a(t)$.

In fact, in Sect. 5 we have found solutions that describe accelerated expansion for the three possible cosmological models of the universe. Namely, spherical expansion $(k=1)$, flat expansion $(k=0)$ and hyperbolic expansion $(k=-1)$ when the constant $\alpha$ is greater than zero. This means that the Einstein-Chern-Simons field equations have as one of their solutions an universe in accelerated expansion. This result allows us to conjecture that these solutions are compatible with the era of dark energy and that the energy-momentum tensor for the field $h^{a}$ corresponds to a form of positive cosmological constant. We have also shown that the EChS field equations have solutions, which allows us to identify the

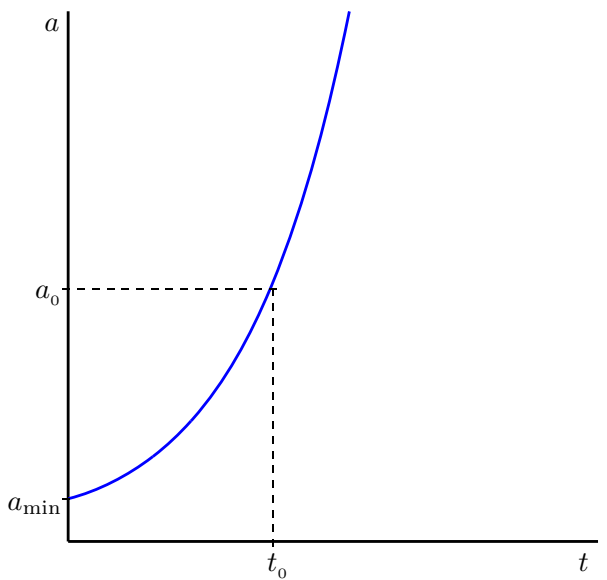

Fig. 14 Solution of $\dot{a}=\sqrt{A a^{2}\left(1-\sqrt{1+\frac{a_{\text {min }}^{4}}{a^{4}}}\right)-k}$ with $A>0$, $k=1$, and $\dot{a}_{0}>\dot{a}_{\text {cri }}$

Table 8 Expanding universe solutions for scale factor of a closed space $k=1$ with $\alpha>0$, where $a_{\min }=\sqrt[4]{\frac{\kappa 5 \alpha l^{2} \rho_{0}}{3}} a_{0}, a_{\max }=\sqrt{\frac{3 \alpha l^{2} k^{2}+\kappa_{5} \rho_{0} a_{0}^{4}}{6 k}}$, $\omega=\sqrt{\frac{2}{\alpha l^{2}}}, \phi=\operatorname{arcos} h\left(\frac{\omega}{\sqrt{k}} a_{0}\right), \rho_{\min }=\left(\frac{6 k a_{0}^{2}}{3 \alpha l^{2} k^{2}+\kappa_{5} \rho_{0} a_{0}^{4}}\right)^{2} \rho_{0}$, $\rho_{\max }=\frac{3}{\kappa_{5} \alpha l^{2}}$ and $\dot{a}_{\text {cri }}=\sqrt{\sqrt{\frac{\kappa_{5} \rho_{0}}{3 \alpha l^{2}}} a_{0}^{2}-k}$. A decelerated solution describes an expanding universe, which then stops the expansion and contracts until the scale factor reaches a minimum $a_{\min }>0$, in a finite time $t_{\max }$

\begin{tabular}{lll}
\hline & Accelerated & Decelerated \\
\hline$a$ & $a_{\min } \leq a$ & $a_{\min } \leq a \leq a_{\max }$ \\
$a(t \rightarrow \infty)$ & $\sim \cos h\left(\omega\left(t-t_{0}\right)+\phi\right)$ & - \\
$\rho \sim \frac{1}{a^{4}}$ & $0<\rho \leq \rho_{\max }$ & $\rho_{\min } \leq \rho \leq \rho_{\max }$ \\
$\dot{a}$ & $\dot{a}_{\mathrm{cri}}<\dot{a}$ & $-\dot{a}_{\mathrm{cri}}<\dot{a}<\dot{a}_{\mathrm{cri}}$ \\
$\dot{a}(t \rightarrow \infty)$ & $\sim \sin h\left(\omega\left(t-t_{0}\right)+\phi\right)$ & - \\
\hline
\end{tabular}

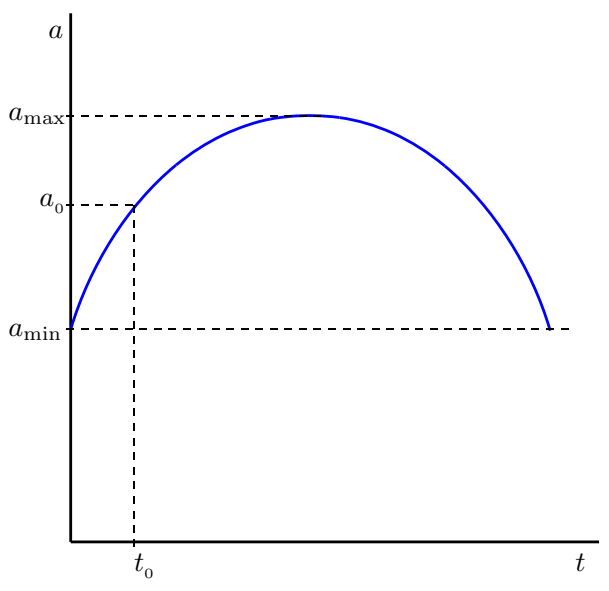

Fig. 15 Solution of $\dot{a}=\sqrt{A a^{2}\left(1-\sqrt{1-\frac{a_{\text {min }}^{4}}{a^{4}}}\right)-k}$ with $A>0$, $k=1$, and $\dot{a}_{0}<\dot{a}_{\text {cri }}$ 
Table 9 Expanding universe solutions with Big Crunch for the scale factor of a closed space $k=1$ with $\alpha<0$, where $a_{\max }=\sqrt{\frac{3 \alpha l^{2} k^{2}+\kappa_{5} \rho_{0} a_{0}^{4}}{6 k}}, \rho_{\min }=\left(\frac{6 k a_{0}^{2}}{3 \alpha l^{2} k^{2}+\kappa_{5} \rho_{0} a_{0}^{4}}\right)^{2} \rho_{0}$, and $\dot{a}_{\max }=$ $\sqrt{\sqrt{-\frac{\kappa_{5} \rho_{0}}{3 \alpha l^{2}}} a_{0}^{2}-k}$. This solution describes an expanding universe, which then stops the expansion and contracts until a Big Crunch, in a finite time $t_{\max }$

\begin{tabular}{ll}
\hline & Decelerate \\
\hline$a$ & $a \leq a_{\max }$ \\
$a(t \rightarrow \infty)$ & - \\
$\rho \sim \frac{1}{a^{4}}$ & $\rho_{\min } \leq \rho$ \\
$\dot{a}$ & $-\dot{a}_{\max }<\dot{a}<\dot{a}_{\max }$ \\
$\dot{a}(t \rightarrow \infty)$ & - \\
\hline
\end{tabular}

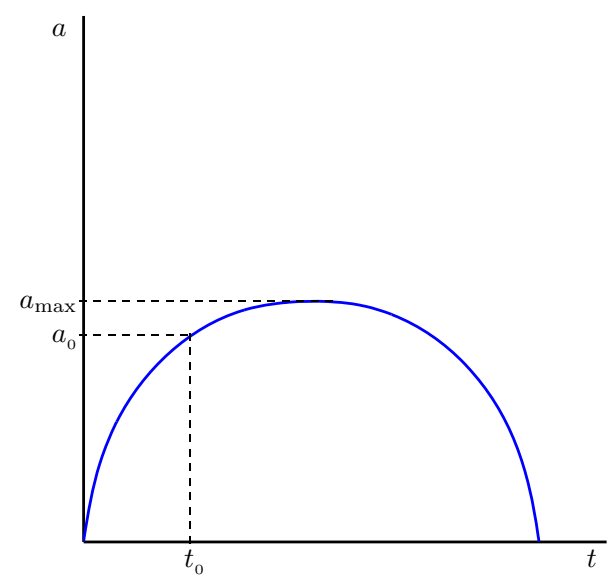

Fig. 16 Solution of $\dot{a}=\sqrt{A a^{2}\left(1-\sqrt{1-\frac{B a_{0}^{4}}{A a^{4}}}\right)-k}$ with $A<0$, $k=1$, and $\dot{a}_{0}<\dot{a}_{\max }$

energy-momentum tensor for the field $h^{a}$ with a negative cosmological constant (Figs. 17, 18, 19, 20, 21, 22, 23, 24).

On the other hand, in Sect. 6 we have found a family of solutions for the era of matter. In the case $k=-1$ (open universe), the solutions correspond to (i) an accelerated expansion $(\alpha>0)$ with a minimum scale factor at initial time that, when time goes to infinity, the scale factor behaves as a hyperbolic sine function; (ii) a decelerated expansion $(\alpha<0)$, with a Big Crunch in a finite time $t_{\max }$, and (iii) a couple of solutions without accelerated expansion, whose scale factor tends to a constant value. In the case $k=0$ (flat universe), the solutions describe (i) an accelerated expansion whose scale factor behaves as an exponential function when time grows and starts from a minimum value, and (ii) a couple of solutions with decelerated expansion whose scale factor tends to square root function. In the case $k=1$ there is found only one solution for a closed universe in expansion, which behaves as a hyperbolic cosine function when time grows and starts from a minimum value. However, there are two

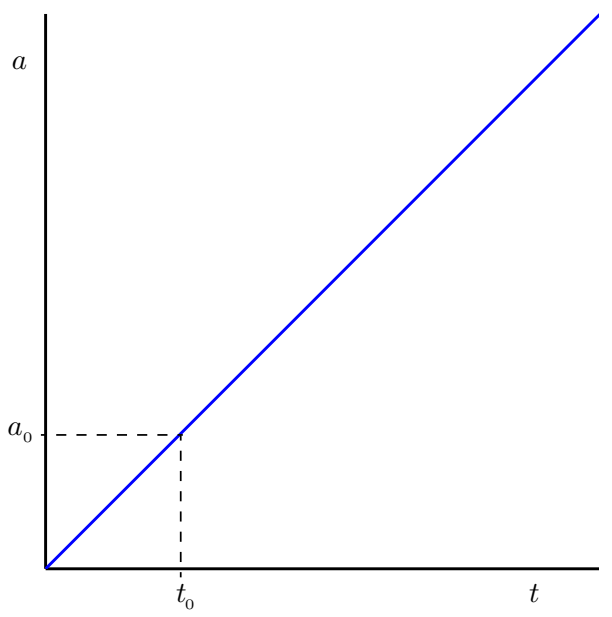

Fig. 17 Graph of $a(t)=\sqrt{-k}\left(t-t_{0}\right)+a_{0}(k=-1)$

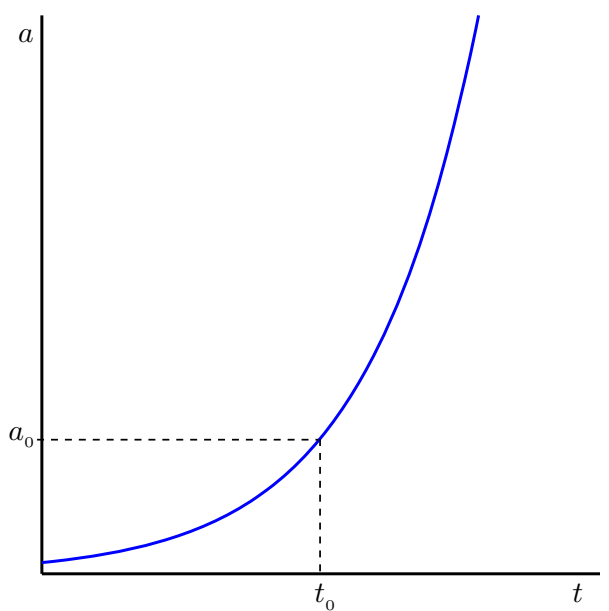

Fig. 18 Graph of $a(t)=a_{0} \exp \left(\sqrt{\frac{2}{\alpha l^{2}}}\left(t-t_{0}\right)\right)$

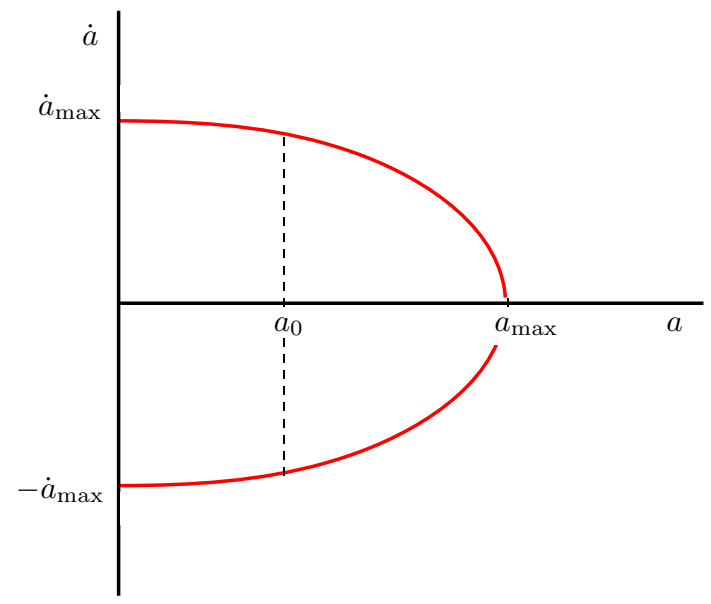

Fig. 19 Phase space for $A<0$ and $k=-1$ with "+" sign

contracting universe solutions, both ending in a finite time. One ends with a minimum value $a_{\min }$, when $\alpha$ is positive, and the other ends with a Big Crunch, when $\alpha$ is negative. 


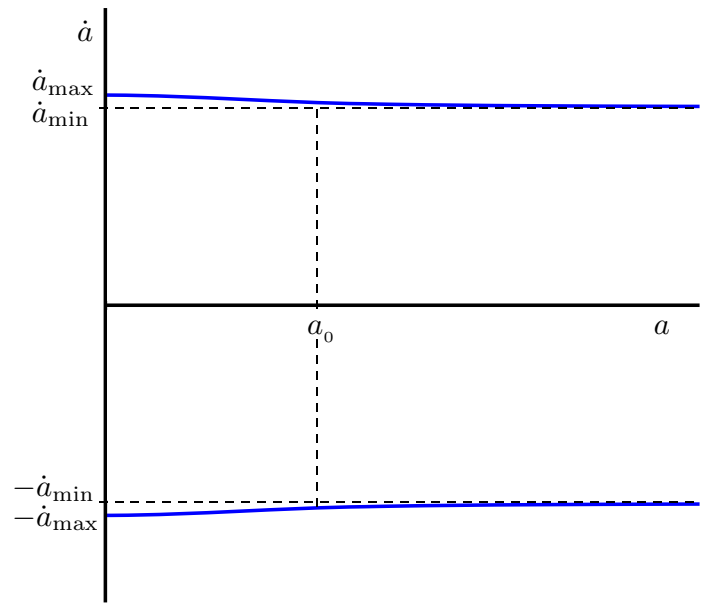

Fig. 20 Phase space for $A<0$ and $k=-1$ with "-_" sign

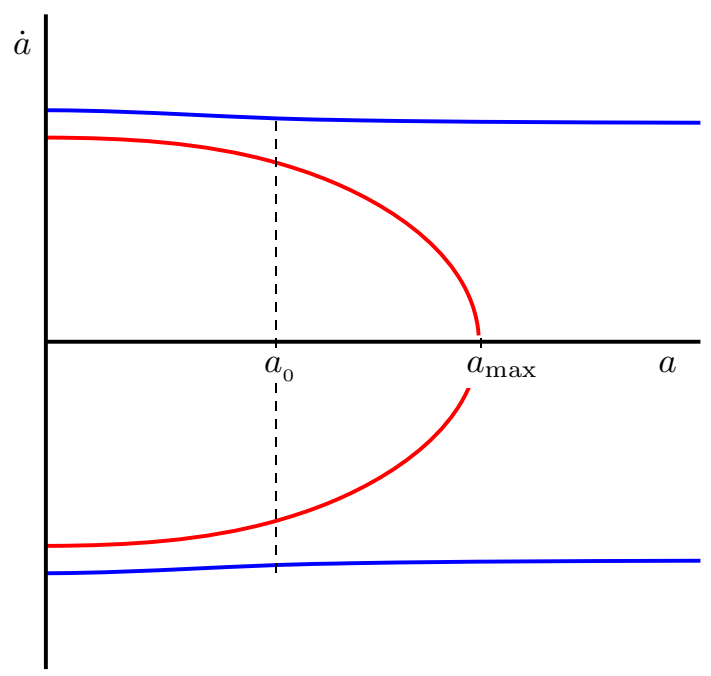

Fig. 21 Phase space for $A<0$ and $k=-1$. Comparison between phase space with "+" sign (Fig. 19) and "-" sign (Fig. 20)

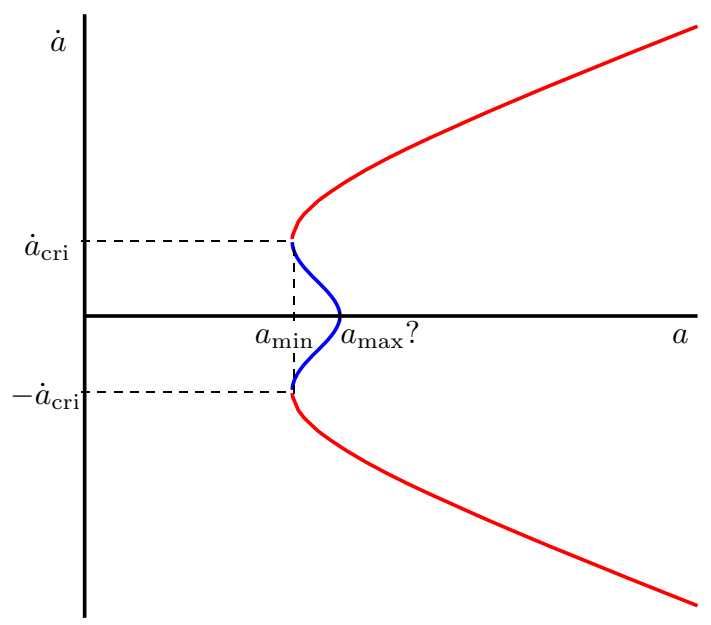

Fig. 22 Phase space for $A>0$ and $k=1$

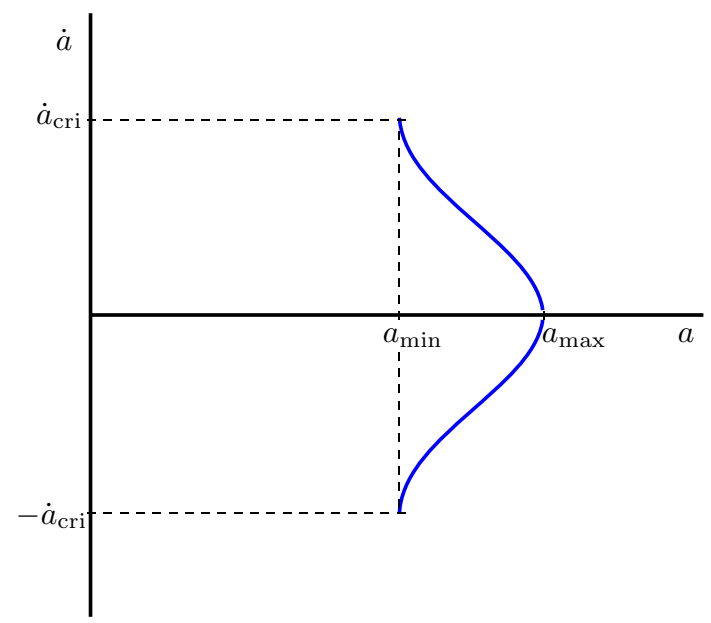

Fig. 23 Phase space for $A>0$ and $k=1$ with "-_ sign

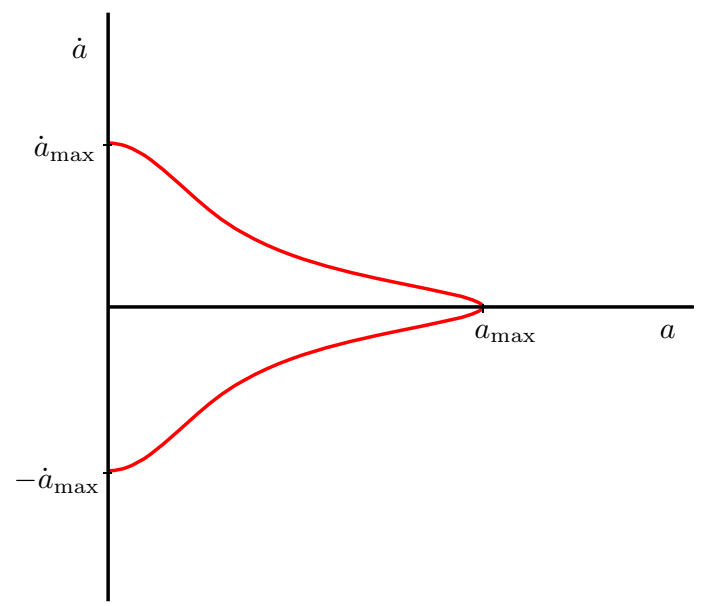

Fig. 24 Phase space for $A<0$ and $k=1$ with "-" sign

In summary, we have found some solutions for the field equations, which were obtained from a Lagrangian for the Chern-Simons gravity theory, studied in Ref. [1]. One problem with these solutions is that they are valid only in a fivedimensional space.

A connection between five-dimensional spacetimes and the four-dimensional universe could be accomplished by using a procedure, based on the Kaluza-Klein theory, known as dynamic compactification $[12,13]$. The method consists in considering a spacetime metric in which the scale factor of the compact space evolves as an inverse power of the radius of the observable universe. In fact the metric can be written in a convenient way so that it can achieve the compactness of the fifth dimension. Following Refs. $[12,13]$ we could consider the five-dimensional metric

$$
\begin{aligned}
\mathrm{d} s^{2}= & -\mathrm{d} t^{2}+a^{2}(t)\left[\left(\mathrm{d} x^{1}\right)^{2}+\left(\mathrm{d} x^{2}\right)^{2}+\left(\mathrm{d} x^{3}\right)^{2}\right] \\
& +b^{2}(t) \mathrm{d} x^{2}
\end{aligned}
$$


and then consider the case when the scale factor $b(t)$ is given by

$b(t)=\frac{1}{a^{n}}, \quad n>0$,

where the parameter $n$ must be positive for dynamical compactification to take place.

Substituting (189) into the metric (188) we have

$$
\begin{aligned}
\mathrm{d} s^{2}= & -\mathrm{d} t^{2}+a^{2}(t)\left[\left(\mathrm{d} x^{1}\right)^{2}+\left(\mathrm{d} x^{2}\right)^{2}+\left(\mathrm{d} x^{3}\right)^{2}\right] \\
& +\frac{\mathrm{d} x^{2}}{a^{2 n}(t)} .
\end{aligned}
$$

Therefore $b$ gets smaller as the radius of our universe $a$ becomes bigger.

It is possible to conjecture that the dynamic compactification procedure could lead, in a certain limit, to the usual results of the four-dimensional General Relativity (work in progress).

It should be noted that this compactification procedure (dynamic compactification) cannot be directly implemented in the theory, because this gravity theory is based on a ChernSimons Lagrangian.

In Ref. [14], subsequently Ref. [15-17], and most recently Ref. [18] it was pointed out that Chern-Simons theories are connected with some even-dimensional structures known as gauged Wess-Zumino-Witten ( $g W Z W)$ terms. In Refs. [19-21] it was shown that a five-dimensional Chern-Simons action invariant under the generalized Poincaré algebra $\mathfrak{B}_{5}$ induces a gauged Wess-Zumino-Witten term containing the four-dimensional Einstein-Hilbert action.

Acknowledgments This work was supported in part by FONDECYT Grants 1130653 and by Universidad de Concepción through DIUC Grant 212.011.056-1.0. Two of the authors (F.G., C.Q.) were supported by grants from the Comisión Nacional de Investigación Científica y Tecnológica CONICYT and from the Universidad de Concepción, Chile. M.C. was supported by Grant FONDECYT 1121030 and by Dirección de Investigación de la Universidad del Bío-Bío through Grants DIUBB 1210072/R and GI1221407/VBC. S.delC. was supported by Grant FONDECYT 1110230 and by Pontificia Universidad Católica de Valparaíso through Grants PUCV 123.710

Open Access This article is distributed under the terms of the Creative Commons Attribution License which permits any use, distribution, and reproduction in any medium, provided the original author(s) and the source are credited.

Funded by $\mathrm{SCOAP}^{3}$ / License Version CC BY 4.0.

\section{Appendix A: Obtaining (35)-(39)}

From (28-32) of Ref. [8] we know that

$48 \alpha_{3}\left(\frac{\dot{a}^{2}+k}{a^{2}}\right)+24 \alpha_{1} l^{2}\left(\frac{\dot{a}^{2}+k}{a^{2}}\right)^{2}=\beta_{1} T_{00}$,

$$
\begin{aligned}
& -24 \alpha_{3}\left[\frac{\ddot{a}}{a}+\left(\frac{\dot{a}^{2}+k}{a^{2}}\right)\right] \\
& -24 \alpha_{1} l^{2} \frac{\ddot{a}}{a}\left(\frac{\dot{a}^{2}+k}{a^{2}}\right)=\beta_{1} T_{11},
\end{aligned}
$$

$24 \alpha_{3} l^{2}\left(\frac{\dot{a}^{2}+k}{a^{2}}\right)^{2}=\beta_{2} T_{00}^{(h)}$,

$-24 \alpha_{3} l^{2} \frac{\ddot{a}}{a}\left(\frac{\dot{a}^{2}+k}{a^{2}}\right)=\beta_{2} T_{11}^{(h)}$,

$24 \alpha_{3} l^{2}\left(\frac{\dot{a}^{2}+k}{a^{2}}\right)\left[(g-f) \frac{\dot{a}}{a}+\dot{g}\right]=0$

where

$h^{0}=f(t) e^{0}$

$h^{p}=g(t) e^{p}, \quad p=1, \ldots, 4$.

In this article we have considered $\beta_{1}=\beta_{2}=\kappa$. Making this replacement in (191)-(197) and dividing it by $8 \alpha_{3}$ we have

$6\left(\frac{\dot{a}^{2}+k}{a^{2}}\right)+\left(\frac{\alpha_{1}}{\alpha_{3}}\right)\left[3 l^{2}\left(\frac{\dot{a}^{2}+k}{a^{2}}\right)^{2}\right]=\left(\frac{\kappa}{8 \alpha_{3}}\right) T_{00}$,

$-8\left[\frac{\ddot{a}}{a}+\left(\frac{\dot{a}^{2}+k}{a^{2}}\right)\right]$

$-\left(\frac{\alpha_{1}}{\alpha_{3}}\right)\left[3 l^{2} \frac{\ddot{a}}{a}\left(\frac{\dot{a}^{2}+k}{a^{2}}\right)\right]=\left(\frac{\kappa}{8 \alpha_{3}}\right) T_{11}$,

$3 l^{2}\left(\frac{\dot{a}^{2}+k}{a^{2}}\right)^{2}=\left(\frac{\kappa}{8 \alpha_{3}}\right) T_{00}^{(h)}$,

$-3 l^{2} \frac{\ddot{a}}{a}\left(\frac{\dot{a}^{2}+k}{a^{2}}\right)=\left(\frac{\kappa}{8 \alpha_{3}}\right) T_{11}^{(h)}$,

$3 l^{2}\left(\frac{\dot{a}^{2}+k}{a^{2}}\right)\left[(g-f) \frac{\dot{a}}{a}+\dot{g}\right]=0$.

Consider now the definition of the constants of Sect. 2:

$\kappa_{5}=\frac{\kappa}{8 \alpha_{3}}, \quad \alpha=-\frac{\alpha_{1}}{\alpha_{3}}$.

With these constants, (198)-(202) take the form

$6\left(\frac{\dot{a}^{2}+k}{a^{2}}\right)-\alpha\left[3 l^{2}\left(\frac{\dot{a}^{2}+k}{a^{2}}\right)^{2}\right]=\kappa_{5} T_{00}$, 
$-8\left[\frac{\ddot{a}}{a}+\left(\frac{\dot{a}^{2}+k}{a^{2}}\right)\right]+\alpha\left[3 l^{2} \frac{\ddot{a}}{a}\left(\frac{\dot{a}^{2}+k}{a^{2}}\right)\right]=\kappa_{5} T_{11}$,

$3 l^{2}\left(\frac{\dot{a}^{2}+k}{a^{2}}\right)^{2}=\kappa_{5} T_{00}^{(h)}$,

$-3 l^{2} \frac{\ddot{a}}{a}\left(\frac{\dot{a}^{2}+k}{a^{2}}\right)=\kappa_{5} T_{11}^{(h)}$,

$3 l^{2}\left(\frac{\dot{a}^{2}+k}{a^{2}}\right)\left[(g-f) \frac{\dot{a}}{a}+\dot{g}\right]=0$.

Substituting now (206) into the square brackets of (204) and (207) into the square brackets of (205), and passing those terms on the right side of the equations, we find

$$
\begin{aligned}
& 6\left(\frac{\dot{a}^{2}+k}{a^{2}}\right)=\kappa_{5} T_{00}+\kappa_{5} \alpha T_{00}^{(h)}, \\
& -8\left[\frac{\ddot{a}}{a}+\left(\frac{\dot{a}^{2}+k}{a^{2}}\right)\right]=\kappa_{5} T_{11}+\alpha \kappa_{5} T_{11}^{(h)}, \\
& 3 l^{2}\left(\frac{\dot{a}^{2}+k}{a^{2}}\right)^{2}=\kappa_{5} T_{00}^{(h)}, \\
& -3 l^{2} \frac{\ddot{a}}{a}\left(\frac{\dot{a}^{2}+k}{a^{2}}\right)=\kappa_{5} T_{11}^{(h)}, \\
& \left(\frac{\dot{a}^{2}+k}{a^{2}}\right)\left[(g-f) \frac{\dot{a}}{a}+g\right]=0 .
\end{aligned}
$$

Accommodating some signs in (210) and (212), grouping some terms (see (209) and (210)), we have

$$
\begin{aligned}
& 6\left(\frac{\dot{a}^{2}+k}{a^{2}}\right)=\kappa_{5}\left(T_{00}+\alpha T_{00}^{(h)}\right), \\
& 8\left[\frac{\ddot{a}}{a}+\left(\frac{\dot{a}^{2}+k}{a^{2}}\right)\right]=-\kappa_{5}\left(T_{11}+\alpha T_{11}^{(h)}\right), \\
& 3 l^{2}\left(\frac{\dot{a}^{2}+k}{a^{2}}\right)^{2}=\kappa_{5} T_{00}^{(h)}, \\
& 3 l^{2} \frac{\ddot{a}}{a}\left(\frac{\dot{a}^{2}+k}{a^{2}}\right)=-\kappa_{5} T_{11}^{(h)}, \\
& \left(\frac{\dot{a}^{2}+k}{a^{2}}\right)\left[(g-f) \frac{\dot{a}}{a}+g\right]=0 .
\end{aligned}
$$

In Sect. 4 we considered an energy-momentum tensor of the form

$$
\begin{aligned}
\tilde{T}_{\mu \nu}= & T_{\mu \nu}+\alpha T_{\mu \nu}^{(h)} \\
= & \operatorname{diag}(\rho, p, p, p, p) \\
& +\alpha \operatorname{diag}\left(\rho^{(h)}, p^{(h)}, p^{(h)}, p^{(h)}, p^{(h)}\right) \\
= & \operatorname{diag}\left(\rho+\alpha \rho^{(h)}, p+\alpha p^{(h)},\right. \\
& \left.p+\alpha p^{(h)}, p+\alpha p^{(h)}, p+\alpha p^{(h)}\right) \\
= & \operatorname{diag}(\tilde{\rho}, \tilde{p}, \tilde{p}, \tilde{p}, \tilde{p})
\end{aligned}
$$

where

$T_{\mu \nu}=\operatorname{diag}(\rho, p, p, p, p)$,

$T_{\mu \nu}^{(h)}=\operatorname{diag}\left(\rho^{(h)}, p^{(h)}, p^{(h)}, p^{(h)}, p^{(h)}\right)$.

Writing the functions $f$ and $g$ as

$f=h(0), \quad g=h$,

we find that (214)-(218) take the form

$6\left(\frac{\dot{a}^{2}+k}{a^{2}}\right)=\kappa_{5} \tilde{\rho}$,

$3\left[\frac{\ddot{a}}{a}+\left(\frac{\dot{a}^{2}+k}{a^{2}}\right)\right]=-\kappa_{5} \tilde{p}$,

$\frac{3 l^{2}}{\kappa_{5}}\left(\frac{\dot{a}^{2}+k}{a^{2}}\right)^{2}=\rho^{(h)}$,

$\frac{3 l^{2}}{\kappa_{5}} \frac{\ddot{a}}{a}\left(\frac{\dot{a}^{2}+k}{a^{2}}\right)=-p^{(h)}$,

$\left(\frac{\dot{a}^{2}+k}{a^{2}}\right)\left[(h-h(0)) \frac{\dot{a}}{a}+\dot{\varphi}\right]=0$,

which correspond to (35)-(39).

\section{References}

1. F. Izaurieta, P. Minning, A. Pérez, E. Rodríguez, P. Salgado, Phys. Lett. B 678, 213 (2009)

2. F. Izaurieta, E. Rodríguez, P. Salgado, J. Math. Phys. 47, 123512 (2006)

3. F. Izaurieta, A. Perez, E. Rodríguez, P. Salgado, J. Math. Phys. 50, 073511 (2009)

4. J. Zanelli, Lecture Notes on Chern-Simons (super)gravities. 2nd ed. (February 2008) (2005)

5. F. Izaurieta, E. Rodriguez, P. Salgado, Lett. Math. Phys. 80, 127 (2007)

6. A.H. Chamseddine, Phys. Lett. B 233, 291 (1989)

7. A.H. Chamseddine, Nucl. Phys. B 346, 213 (1990)

8. F. Gomez, P. Minning, P. Salgado, Phys. Rev. D 84, 063506 (2011)

9. C.A.C. Quinzacara, P. Salgado, Phys. Rev. D 85, 124026 (2012) 
10. S. Weinberg, Gravitation and cosmology: principles and applications of the general theory of relativity (Wiley, New York, 1972)

11. S. del Campo, JCAP 1212, 005 (2012)

12. K. Andrew, B. Bolen, C. Middleton, Gen. Rel. Grav. 39, 2061 (2007)

13. N. Mohammedi, Phys. Rev. D 65, 104018 (2002)

14. L. Alvarez-Gaumé, P.H. Ginsparg, Ann. Phys. 161, 423 (1985)

15. A. Anabalon, S. Willison, J. Zanelli, Phys. Rev. D 75, 024009 (2007)
16. A. Anabalon, S. Willison, J. Zanelli, Phys. Rev. D 77, 044019 (2008)

17. A. Anabalon, JHEP 0806, 069 (2008)

18. P. Mora, P. Pais, Phys. Rev. D 84, 044058 (2011)

19. P. Salgado, P. Salgado-Rebolledo, O. Valdivia, Phys. Lett. B 728, 99 (2014)

20. P. Salgado, R.J. Szabo, O. Valdivia, Phys. Rev. D 89, 084077 (2014)

21. S. Salgado, F. Izaurieta, N. Gonzalez, G. Rubio, Phys. Lett. B 732, 255 (2014) 